\title{
Analytic baby skyrmions at finite density
}

\author{
Marco Barsanti ${ }^{1, \mathrm{a}}$, Stefano Bolognesi ${ }^{1, \mathrm{~b}}$, Fabrizio Canfora ${ }^{2, \mathrm{c}}$, Gianni Tallarita ${ }^{3, \mathrm{~d}}$ \\ ${ }^{1}$ Department of Physics “E. Fermi”, University of Pisa and INFN, Sezione di Pisa Largo Pontecorvo, 3, Ed. C, 56127 Pisa, Italy \\ ${ }^{2}$ Centro de Estudios Científicos (CECS), Casilla 1469, Valdivia, Chile \\ ${ }^{3}$ Departamento de Ciencias, Facultad de Artes Liberales, Universidad Adolfo Ibáñez, 7941169 Santiago, Chile
}

Received: 3 July 2020 / Accepted: 17 November 2020 / Published online: 28 December 2020

(C) The Author(s) 2020

\begin{abstract}
We study the baby Skyrme model in $(2+1)$ dimensions built on a finite cylinder. To this end, we introduce a consistent ansatz which is able to reduce the complete set of field equations to just one equation for the profile function for arbitrary baryon charge. Many analytic solutions both with and without the inclusion of the effects of the minimal coupling with the Maxwell field are constructed. The baby skyrmions appear as a sequence of rings along the cylinder, leading to a periodic shape in the baryon density. Linear stability and other physical properties are discussed. These analytic gauged baby Skyrmions generate a persistent $U(1)$ current which cannot be turned off continuously as it is tied to the topological charge of the baby Skyrmions themselves. In the simplest non-trivial case of a gauged baby Skyrmion, a very important role is played by the Mathieu equation with an effective coupling constant which can be computed explicitly. These configurations are a very suitable arena to test resurgence in a non-integrable context.
\end{abstract}

\section{Contents}

1 Introduction . . . . . . . . . . . . 1

2 Beyond the spherical hedgehog ansatz . . . . . . . 2

3 Ungauged baby Skyrme model . . . . . . . . . . . . 4

3.1 Kink-dressed topological solitons . . . . . . . . 5

3.2 Energy density . . . . . . . . . . . . 8

3.3 A remark on the stability . . . . . . . . . 9

3.4 The case of potential $V=\frac{1}{2}\left(1-\phi_{3}^{2}\right) \ldots \ldots 11$

4 Gauged baby Skyrmions . . . . . . . . . . . 11

4.1 On the construction of the ansatz for the gauged solitons . . . . . . . . . . 12

\footnotetext{
a e-mail: marco.barsanti@phd.unipi.it

be-mail: stefano.bolognesi@unipi.it (corresponding author)

ce-mail: canfora@cecs.cl

d e-mail: gianni.tallarita@uai.cl
}

4.2 Gauged baby Skyrmions and resurgence . . . 16

4.3 Persistent residual currents . . . . . . . . . 20

5 Conclusions ................. 21

References . . . . . . . . . . . . . . . . 21

\section{Introduction}

The (gauged) baby Skyrme model in (2+1)-dimensions is one of the most interesting models admitting solitons without the huge analytic difficulties present in the $(3+1)$ dimensional case (see [1-10]; for two detailed reviews see [13] and [14]). Due to the fact that the (gauged) baby Skyrme model shares many relevant physical features with its $(3+1)$ dimensional progenitor (see [15-26] and references therein), it has been deeply analyzed as it can shed light on relevant non-perturbative features of a field theory with nontrivial topological sectors. On the other hand, despite the fact that it is simpler than the (gauged) Skyrme model in $(3+1)$-dimensions, it is manifestly a non-integrable system for which tools like the Lax pairs or the bi-Hamiltonian formalism cannot be applied. For these reasons, with few exceptions, most of the multi-solitonic solutions in the baby Skyrme model in $(2+1)$-dimensions have been constructed numerically (see [15-26] and references therein). In the present manuscript we want to construct analytic multisolitonic configurations of the (gauged) baby Skyrme model in (2+1)-dimensions. These enable one to disclose novel nonperturbative features of the theory.

We are especially interested in the following two important issues. Firstly, we would like to understand how finitesize effects affect multi-solitonic configurations. Secondly, we would like to understand the electromagnetic properties of these solitons when finite-size effects cannot be neglected.

The great physical interest of finite density effects arises from the fact that a theoretical description of cold and dense nuclear matter as a function of baryon number density is 
still lacking. In this sense, the (gauged) baby Skyrme model in $(2+1)$-dimensions is a very interesting model which is not integrable (but still easier than the $(3+1)$-dimensions (gauged) Skyrme model) in which one can analyze finite density effects with non-perturbative methods. In contrast to what happens in condensed matter physics, exact analytic results on the phase diagram of the low energy limit of QCD at finite density and low temperatures are extremely rare. The non-perturbative nature of low energy QCD explains why the very complex and interesting structure of its phase diagram (see [28-34] and references therein) is mainly analyzed with numerical and lattice approaches. A remarkable phenomena in the QCD phase diagram (which appears at finite baryon density) is the appearance of ordered structures of solitons (as it happens, for instance, in condensed matter theory with the Larkin-Ovchinnikov-Fulde-Ferrell phase [35-37]). Many results by now support the existence of these ordered structures at finite density (see, for instance, [38-63, 63], and references therein). The very few analytic results have been found either in (1+1)-dimensions [45-51] or when some extra symmetries (such as SUSY) are included (see [15-26] and references therein). That is why it is important to shed more light on these intriguing phenomena as, often, even the numerical approaches are in trouble when the topological charge is very high: the analytic tools developed here below disclose novel features of multi-solitonic solutions in the (gauged) baby Skyrme model in (2+1)-dimensions.

The analytic approach which will be developed here will also shed light on a novel relation between (gauged) multisolitons in the (gauged) baby Skyrme model and resurgence. Resurgence (see [65-71] and references therein) provides us with a concrete hope to make sense of the divergent character of perturbation series in QFT. This approach (using nonperturbative informations related with the non-trivial saddle points of the path integral) works, roughly, as follows: one is instructed to use Borel summation in the complex $g$-plane ( $g$ being a relevant coupling constant of the theory) to give a meaning to the factorially divergent series of perturbation theory. The initial divergent series becomes a finite expression. This expression possesses ambiguities which manifest themselves along suitable lines in the complex $g$-plane. At a first glance, one has just traded one problem for another (maybe even worse). In fact, when the theories one is interested in possess non-perturbative sectors labelled by suitable topological charges, the perturbative expansions in these topologically non-trivial sectors (which, usually, are also factorially divergent) allow a remarkable simplification (as shown for the first time in the physical literature in $[71,72])$. The "Borel ambiguities" arising in the topologically nontrivial sectors exactly cancel those of the perturbative sector: in this way, by including all the non-perturbative sectors on the same footing, one gets a well-defined answer. Starting from the nice results in [73], in recent years this topic has attracted a lot of attention. The theories which have been mostly analyzed from the resurgent point of view have been quantum mechanics, topological strings, and integrable quantum field theory in low dimensions (see [65,75-81] and references therein). One of the main building blocks of the resurgent scheme is a proper analytic understanding of the non-trivial saddle points of the path integral. Hence, it is of fundamental importance to deepen our knowledge of the classical solutions of field theory with non-trivial topological charges. This task is very urgent for non-integrable field theories in which the theoretical tools coming from SUSY and the saturation of the BPS bound are not effective. As explained in the next sections, the framework developed in the present manuscript is useful from a resurgent perspective. Finite density effects are also interesting from this perspective. In particular, how the properties of multi-solitonic configurations depend on the typical size of a compact spatial domain in which these multi-solitonic configurations live, is especially interesting from a resurgent perspective (see [65-73,75-81] and references therein).

In the present paper, the methods of [82-92] will be generalized to construct analytic solutions for baby Skyrmions at finite density. We will organize the paper as follows. In Sect. 2 we begin by discussing the nature of our ansatz. In Sect. 3 we analyse the results of a specific ansatz on ungauged baby skyrme models and in Sect. 4 we show how this can be extended to the gauged case, providing also some links to the topic of resurgence. We will conclude with a discussion of results in Sect. 5.

\section{Beyond the spherical hedgehog ansatz}

The action of the gauged baby Skyrme system is

$$
\begin{aligned}
S= & \int d^{3} x \sqrt{-g}\left[\frac{a_{1}}{2}\left(D_{\mu} \vec{\Phi}\right) \cdot\left(D_{\mu} \vec{\Phi}\right)-a_{0}\left(\frac{1-\vec{\Phi} \cdot \vec{n}}{2}\right)^{k}\right. \\
& -\frac{a_{2}}{4}\left(D_{\mu} \vec{\Phi} \times D_{\nu} \vec{\Phi}\right) \cdot\left(D_{\mu} \vec{\Phi} \times D_{\nu} \vec{\Phi}\right) \\
& \left.-\lambda(\vec{\Phi} \cdot \vec{\Phi}-1)-\frac{1}{4 e^{2}} F_{\mu \nu} F^{\mu \nu}\right]
\end{aligned}
$$

where

$D_{\mu} \vec{\Phi}=\nabla_{\mu} \vec{\Phi}+A_{\mu} \vec{n} \times \vec{\Phi}, \quad F_{\mu \nu}=\nabla_{\mu} A_{\nu}-\nabla_{\nu} A_{\mu}$

$\lambda$ is a Lagrange multiplier implementing the geometric constraint $\vec{\Phi} \cdot \vec{\Phi}=1$ in isospin space, $a_{j}, e$ are coupling constants and $F_{\mu \nu} F^{\mu \nu}$ is the usual Maxwell Lagrangian. In this paper, we use the same notation adopted in $[11,12]$ in which, resclaling the action by an overall constant and rescaling the length scale, we work with dimensionless coordinates $\left\{x^{i}\right\}$. As a result, also the couplings $a_{j}, e$ are dimensionless. It is convenient to parametrize the fields as follows 
$\vec{\Phi} \cdot \vec{\Phi}=1 \Longleftrightarrow \vec{\Phi}=(\sin F \cos G, \sin F \sin G, \cos F)$,

where

$F=F\left(x^{\mu}\right), \quad G=G\left(x^{\mu}\right)$,

and we orient the minimum of the potential in the position

$\vec{n}=(0,0,1)$

In this parametrization the replacement of $\nabla_{\mu} \vec{\Phi}$ with $D_{\mu} \vec{\Phi}$ of the usual partial derivative with the covariant $U(1)$ derivative defined above corresponds to just replace $\nabla_{\mu} G$ with $\left(\nabla_{\mu} G+\right.$ $\left.A_{\mu}\right)$ in the action while $\nabla_{\mu} F$ remains unaltered.

The parametrization in Eqs. (3), (4) is the most general parametrization of a gauged baby Skyrmion. The system is described by two scalar degrees of freedom, as $\vec{\Phi}$ satisfies one scalar constraint, plus the degrees of freedom of the Maxwell field. Moreover, we can always rotate the Isospin vector $\vec{n}$ along the third internal axis. Hence, one can write the field equations either in terms of $\vec{\Phi}$ (which satisfies $\vec{\Phi} \cdot \vec{\Phi}=1$ ) and $A_{\mu}$ or in terms of $F, G$ and $A_{\mu}$ : the two ways to write the field equations are completely equivalent. In order to build a good ansatz, it is better for us to work with $F, G$ and $A_{\mu}$.

With the above parametrization, the gauged baby Skyrme action reads

$S=\int d^{3} x \sqrt{-g}\left(\mathcal{L}^{\prime}-\frac{1}{4 e^{2}} F_{\mu \nu} F^{\mu \nu}\right)$

where

$$
\begin{aligned}
\mathcal{L}^{\prime}= & \frac{a_{1}}{2}\left(\nabla_{\mu} F \nabla^{\mu} F+\sin ^{2} F\left(\nabla_{\mu} G+A_{\mu}\right)\left(\nabla^{\mu} G+A^{\mu}\right)\right) \\
& -\frac{a_{2}}{2} \sin ^{2} F\left[\left(\nabla_{\mu} F \nabla^{\mu} F\right)\left(\nabla_{\nu} G+A_{\nu}\right)\left(\nabla^{\nu} G+A^{v}\right)\right. \\
& \left.-\left(\left(\nabla_{\mu} F\right)\left(\nabla^{\mu} G+A^{\mu}\right)\right)^{2}\right]-V,
\end{aligned}
$$

where

$V=a_{0}\left(\frac{1-\cos F}{2}\right)^{k}=a_{0} \sin ^{2 k}\left(\frac{F}{2}\right)$.

Obviously, the Lagrangian in Eqs. (5) and (6) is equivalent to the one in Eqs. (1) and (2). In other words, the Lagrangian in Eqs. (5) and (6) correctly describes the two scalar degrees of freedom of $\vec{\Phi}$ as well as the degree of freedom associated to the gauge potential $A_{\mu}$. The gauged baby Skyrme field equations can be obtained by taking the variation of the action with respect to $F, G$ and $A_{\mu}$.

The easiest way to take into account finite-density effects is to introduce the cylinder flat metric

$d s^{2}=-d t^{2}+d r^{2}+L^{2} d \phi^{2}$

where $\phi$ is an angular coordinate with range

$0 \leq \phi \leq 2 \pi$ and $L$ is the compactification radius. The range of the coordinate $r$ will be chosen as follows:

$0 \leq r \leq R$

where $R$ represents the height of the cylinder. Physical constraints on $R$ will appear when discussing the boundary conditions.

Given the metric (8) and requiring the positivity of the energy, we choose for the coefficients $a_{1}, a_{2}$ and $a_{0}$ the conditions

$a_{1}<0, \quad a_{2} \geq 0, \quad a_{0} \geq 0$.

We remind that due to our choice the coefficients (11) and the coordinates (8) are dimensionless.

The most difficult task is to find a good ansatz which has non-trivial topological charge and, at the same time, allows for a multi-peaks structure in the energy density without making the field equations impossible to solve analytically.

The topological charge of the configuration for $A_{\mu}=0$ is

$B=\frac{1}{4 \pi} \int_{\Sigma} \rho_{B}$,

where $\Sigma$ is a two-dimensional space-like surface (for example $t=$ const surfaces) and, in terms of $F$ and $G$, the topological density $\rho_{B}$ reads

$\rho_{B}=\frac{1}{4 \pi}(\sin F) d F \wedge d G$,

so that a necessary condition in order to have non-trivial topological charge is

$d F \wedge d G \neq 0$.

From the geometrical point of view the above condition (which simply states that $F$ and $G$ must be two independent functions) can be interpreted as saying that such two functions "fill a two-dimensional spatial volume" at least locally. On the other hand, such a condition is not sufficient in general. One has also to require that the spatial integral of $\rho_{B}$ must be a non-vanishing integer:

$\int_{\Sigma} \rho_{B} \in \mathbb{Z}$

Usually, this second requirement allows one to fix some of the parameters of the ansatz.

Following the strategy of [84-88] and [92], one can consider the following ansatz

$F=F(r), \quad G=p \phi-\omega t, \quad p \in \mathbb{N}$.

Note that the baby Skyrme field depends on the function $G$ only through $\sin G$ and $\cos G$ so that this solution is periodic in time. The periodic time dependence in Eq. (16) is a natural way to avoid Derrick's no-go theorem on the existence of solitons in non-linear scalar field theories and corresponds to 
a time-periodic ansatz such that the energy density of the configuration is still static. The present ansatz defined in Eqs. (3), (16) has exactly this property. We will consider first in Sect. 3 static solutions in which $\omega=0$. The importance of introducing a time-dependence will be apparent in Sect. 4 on gauged baby Skyrmions where $\omega$ will have a specific relation with $p$.

When the gauge potential is non-vanishing $A_{\mu} \neq 0$ an extra term must be included in the definition of the topological charge in order to ensure both the topological and the gauge invariance. In analogy with the gauged Skyrme model in (3+1)-dimensions (see [4] and [106]) the topological charge of the gauged baby Skyrme model in $(2+1)$ dimensions is given by:

$$
\begin{aligned}
\rho_{B} & =\frac{1}{4 \pi}\left[\sin F d F \wedge(d G+A)+\frac{F}{2}(1-\cos F)\right], \\
A & =A_{\mu} d x^{\mu} F=F_{\mu \nu} d x^{\mu} \wedge d x^{\nu} .
\end{aligned}
$$

Indeed, it is a direct computation to check that the topological charge in Eqs. (17) coincides with the usual definition of topological charge of the gauged baby Skyrme model in the literature [15-26]. The definition (17) can be decomposed into the sum of the ungauged topological density plus a total derivative

$\rho_{B}=\frac{1}{4 \pi}\left[\sin F \partial_{\mu} F \partial_{\nu} G+\partial_{\mu}\left(A_{\nu}(1-\cos F)\right)\right] d x^{\mu} \wedge d x^{\nu}$.

From this form, it is evident that the gauge component of the topological charge is a boundary term.

\section{Ungauged baby Skyrme model}

In this section we will consider first the baby Skyrme model without the $U(1)$ gauge field ( $A_{\mu}=0$ or formally $e \rightarrow 0$ ). The variation of the baby Skyrme action with respect to $F$ leads to the equation of motion

$$
\begin{aligned}
0= & -\square F+\frac{\sin (2 F)}{2} \nabla_{\mu} G \nabla^{\mu} G \\
& -c_{3} \frac{\sin (2 F)}{2}\left[\left(\nabla_{\mu} F \nabla^{\mu} F\right)\left(\nabla_{\nu} G \nabla^{\nu} G\right)-\left(\nabla_{\mu} F \nabla^{\mu} G\right)^{2}\right] \\
& +c_{3} \nabla_{\mu}\left[\sin ^{2}(F)\left[\left(\nabla_{\nu} G \nabla^{\nu} G\right) \nabla^{\mu} F-\left(\nabla_{\nu} F \nabla^{\nu} G\right) \nabla^{\mu} G\right]\right] \\
& -\frac{1}{a_{1}} \frac{\partial}{\partial F} V .
\end{aligned}
$$

The variation of the baby Skyrme action with respect to $G$ leads to the equation of motion

$$
\begin{aligned}
0= & -\sin ^{2}(F) \square G-\sin (2 F) \nabla_{\mu} F \nabla^{\mu} G \\
& -c_{3} \nabla_{\mu}\left[\operatorname { s i n } ^ { 2 } ( F ) \left[\left(\nabla_{\nu} F \nabla^{\nu} G\right) \nabla^{\mu} F\right.\right. \\
& \left.\left.-\left(\nabla_{\nu} F \nabla^{\nu} F\right) \nabla^{\mu} G\right]\right] .
\end{aligned}
$$

In the above equations $c_{3}=\frac{a_{2}}{a_{1}}$.

The above choice of the ansatz (16) the field equations simplifies dramatically (as shown below) due to the fact that, with the function $G$ in Eq. (16),

$\nabla_{\mu} G \nabla^{\mu} G=\frac{p^{2}}{L^{2}}=K$,

$\nabla_{\mu} G \nabla^{\mu} F=0$.

One can check with a direct computation that the ansatz defined in Eq. (16) does indeed reduce the full system of field equations to just one integrable equation for $F$.

With such ansatz, the topological charge density (13) reads

$\rho_{B}=\frac{p}{4 \pi} \sin F\left(\partial_{r} F\right) d r \wedge d \phi$.

The above ansatz satisfies the first condition to be topologically non-trivial in Eq. (14) as $F$ and $G$ in Eq. (16) are independent. The boundary condition on $F$ and the corresponding baby Baryon charge are

$$
\begin{aligned}
& F(0)=0, \quad F(R)=\pi(1+2 n) \quad n \in \mathbb{N} \quad \Longrightarrow \quad B=p \\
& F(0)=0, \quad F(R)=2 m \pi \quad m \in \mathbb{N} \Longrightarrow B=0
\end{aligned}
$$

The integers $n$ and $m$ appearing in Eqs. (23), (24) do not appear in the topological charge but, together with $p$, they are relevant to classify the map. The meaning of these integers needs some comments. If the integer $p$ corresponds to the number of windings from the angular variable $\theta$ of the cylinder into the azimuthal angle $G$ of the sphere, the integers $n$ or $m$ are connected with the "polar" windings of the sphere. A way to visualize the map (16) with the boundary conditions (23) or (24) can be thought following two steps. Firstly, we map the circumference of the cylinder at $r=0$ p-times into the $G$ angular coordinate at $F=0$. Then, we extend this map along the r-direction on the cylinder wrapping the sphere along the polar $F$-coordinate. If we reach the value of $F=\pi$ at $r=R$ (the case $n=0$ in (23)), the sphere is completely wrapped and the topological charge is $B=p$. However, if $F$ goes over $\pi$, the map starts to unwind the sphere along the $F$-direction till $F=2 \pi$ at $r=R$. In this case $(m=1$ in (23)) the sphere is wrapped and unwrapped along the $F$-direction so that the topological charge is $B=0$. Increasing again the value of $F$ the map alternates the windings and unwindings of the target space along the "polar" direction. For this reason, if $F$ at $r=R$ is fixed to be an even number of $\pi$, the topological charge will always be zero; otherwise in the odd case the total sum of positive "polar" windings will be always one and the total topological charge $B=p$, regardless of $n$.

A more rigorous mathematical proof of (23) and (24) can be performed after some comments on the expression (13). 
For our aim, it is necessary that the topological density $\rho_{B}$ is well defined for every interval of coordinates, with particular attention when $F$ exceeds the value of $\pi$. As a check, we expect a change of sign in the measure of the sphere (13) when $F$ passes from the interval $[0, \pi]$ to $[\pi, 2 \pi]$ (if we keep the same orientation along the $G$-direction). This change of sign must reflect the two different directions in the path from the north pole to the south pole of the sphere and, consistently, it is provided by the presence of $\sin F$ for every interval of $F$. After these considerations, we can calculate explicitly the topological charge

$$
\begin{aligned}
B & =\frac{p}{4 \pi} \int_{0}^{2 \pi} d \phi \int_{0}^{R} \sin F\left(\partial_{r} F\right) d r \\
& =\frac{p}{2}[-\cos F]_{F(0)}^{F(R)} \\
& =\left\{\begin{array}{l}
\frac{p}{2}(-\cos (\pi+2 n \pi)+\cos (0))=p \\
\frac{p}{2}(-\cos (2 m \pi)+\cos (0))=0
\end{array}\right.
\end{aligned}
$$

as anticipated in (23) and (24).

Nevertheless, even if $n$ and $m$ do not contribute to the total topological charge, they play an important role. As we discussed before, the integers $n$ or $m$ classify the number of windings and unwindings along the "polar" coordinate of the sphere. Therefore, even without knowing the exact form of the functions $F$ and $G$, we can predict that these solutions will oscillate from positive to negative topological charge density. The number of these oscillations will depend on $n$ and $m$ (it will be $2 n+1$ for the case (23) and $2 m$ for (24)). These integers thus indicate the presence of soliton-antisoliton (baryonantibaryon) inside a solution of total charge $B=p$. We call it a topological soliton with charge $p$ "dressed up" with $2 n+1$ (or $2 m$ ) kinks. In a sense, the integers $n$ and $m$ can be thought of a second "charge" of the configuration.

\subsection{Kink-dressed topological solitons}

When one plugs the ansatz in Eq. (16) (remember that in this section the ansatz is static) into the baby Skyrme field equations in Eqs. (19) and (20), they reduce to only one integrable equation for $F(r)$ :

$$
\begin{aligned}
0= & \left(1-K c_{3} \sin ^{2}(F)\right) F^{\prime \prime}-K c_{3} \frac{\sin (2 F)}{2}\left(F^{\prime}\right)^{2} \\
& +\frac{\partial}{\partial F}\left[\frac{V}{a_{1}}-\frac{K}{2} \sin ^{2}(F)\right] .
\end{aligned}
$$

A useful check of consistency of this equation can be provided coming back to dimensional coordinates, in which $\{r, L \phi\}$ have now the dimension of length. As a consequence, the coefficients $c_{3}, K$ and $\left(a_{0} / a_{1}\right)$ have now the dimensions of $\left[c_{3}\right]=l^{2},[K]=l^{-2}$ and $\left[a_{0} / a_{1}\right]=l^{-2}$, where $l$ denotes the length. The different powers in unit of length of the ratios $c_{3}=\left(a_{2} / a_{1}\right)$ and $\left(a_{0} / a_{1}\right)$ follow the different number of derivatives in the action (1). A quick calculation shows how all the terms of (26) posses the same dimensions and then the equation is consistent. The same check leads to the same result in the rest of the paper and then we can come back to the easiest choice of dimensionless coordinates adopted from the beginning.

When the exponent $k$ in the potential (7) is an integer, some of the formulas below simplify (see the next discussion). It is a trivial computation to see that the field equation for $G$ in Eq. (20) is identically satisfied with the ansatz in Eq. (16). One can write Eq. (26) as follows:

$\partial_{r}\left[Y(F) \frac{\left(\partial_{r} F\right)^{2}}{2}+W(F)\right]=0$,

and then

$Y(F) \frac{\left(\partial_{r} F\right)^{2}}{2}+W(F)=E_{0}$

where

$\begin{aligned} Y(F) & \equiv 1-K c_{3} \sin ^{2}(F), \\ W(F) & \equiv \frac{V}{a_{1}}-\frac{K}{2} \sin ^{2}(F) .\end{aligned}$

So Eq. (28) becomes ${ }^{1}$

$\frac{d F}{\eta\left(F, E_{0}\right)}=d r$

where

$\eta\left(F, E_{0}\right) \equiv \frac{\left[2\left(E_{0}-W(F)\right)\right]^{1 / 2}}{Y(F)^{1 / 2}}$,

and $E_{0}$ is a constant to be fixed by the boundary conditions (23), (24) integrating equation (30) as

$\int_{0}^{F(R)} \frac{d F}{\eta\left(F, E_{0}\right)}=R$.

Using the ansatz in Eq. (16) all the baby Skyrme field equations in Eqs. (19) and (20) are satisfied if Eq. (28) is satisfied. The integral equation (32) represents a sort of state equation for the system that, once given the length $R$ and the number of "bumps" (with $F(R)$ ), fixes uniquely the value of $E_{0}$. In other words, the profile function $F(r)$ can be found not only solving the second order differential equation (19) with the two boundary conditions in (23) or in (24) but even solving the first order equation (28) with a single boundary condition and the constant $E_{0}$.

The choice of the cylindrical metric (11) is useful to take into account finite-density effects and to build periodic solutions. However, if along the $\phi$-direction the periodicity is given by the geometry of the cylinder, along the $r$-direction such a periodicity must be imposed to obtain a crystal-like

\footnotetext{
1 The positive sign of the square root of $\left(\partial_{r} F\right)^{2}$ has been chosen.
} 
structure. This is the case of the periodic boundary conditions (24). Moreover, a crystal-configuration to be physical needs the continuity of the baryon density and the energy density. This condition can be obtained imposing the periodicity in the first derivative of the field, that in this case reads only

$$
\left.\partial_{r} F\right|_{r=0}=\left.\partial_{r} F\right|_{r=R} .
$$

The same condition for $G$ and the periodicity of the $\phi$-first derivative is automatically given by the the form of the ansatz (16). It is easy to check that (33) is always verified when we choose periodic boundary conditions (24). Indeed, in Eqs. (30), (31) the first derivative of $F$ results equal to the $2 \pi$ periodic function $\eta(F)$ that contains only powers of $\sin F$ and $\cos F$. As a result, for every integer $m$ of (24) the condition (33) is respected. To summarize, the condition (24) will lead to a physical crystal-like solution that can be extended periodically in the $\mathbb{R}^{2}$ plane. Differently, the condition (23) generates non-trivial topological solutions that live on the finite space and that represent an example of ordered multisolitons located in a finite box.

In Figs. 1, 2, 3, we show the plots of the profile function $F(r)$ and the topological charge density $\rho_{B}(r)$ for different

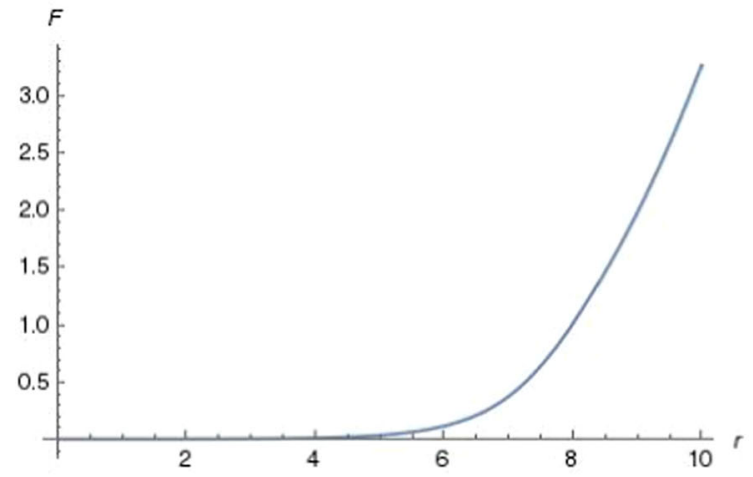

(a) $F(r)$

Fig. 1 Solution with $n=0$

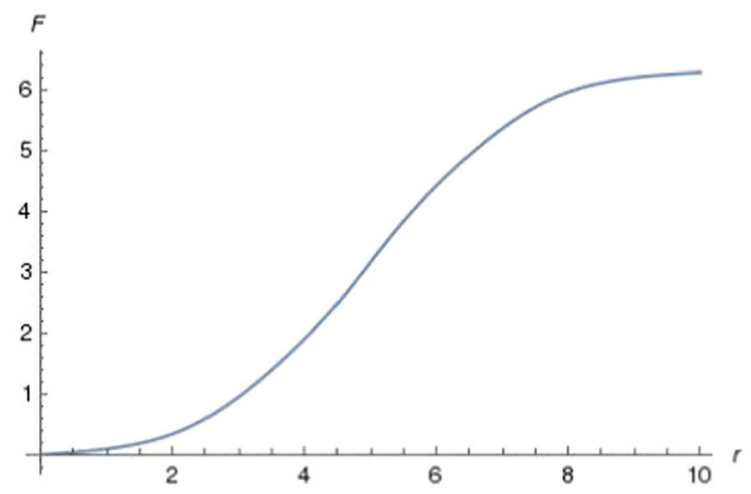

(a) $F(r)$ baby Skyrmion solutions for different boundary conditions, respectively $n=0, m=1, n=1$ as defined in (23) and (24). The set of parameters used for all the plots are

$$
\begin{array}{rlrl}
a_{0} & =1 & & \\
a_{1} & =-1 & L=1 & p=1 \\
a_{2} & =1 & R=10 & K=1 \\
k & =1 & &
\end{array}
$$

Using equation (28) to solve the profile function $F(r)$ with the fixed parameters (34), the constants $E_{0}$ for the solutions $n=0, m=1, n=1$ are respectively

$$
\begin{array}{ll}
n=0 & E_{0}=0 \\
m=1 & E_{0}=0.00268 \\
n=1 & E_{0}=0.1181 .
\end{array}
$$

The plots obtained with Eqs. (19) or (28) are totally equivalent.

As anticipated in this section and as shown in the plots, the solutions with $n>0$ or $m>0$ have positive and negative oscillations in the topological charge density. The number

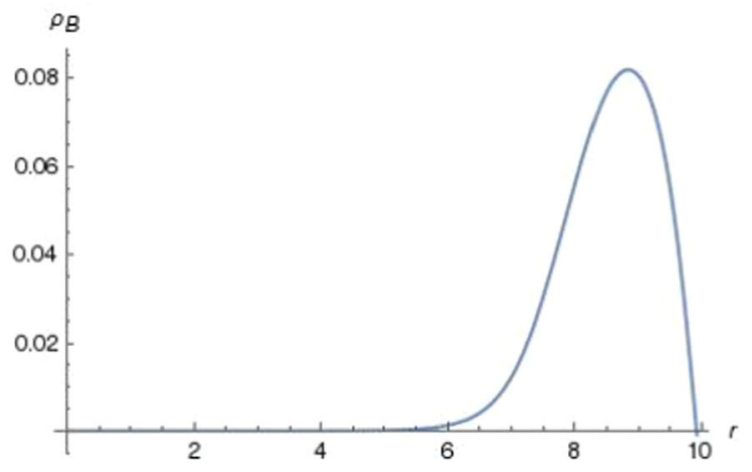

(b) $\rho_{B}(r)$

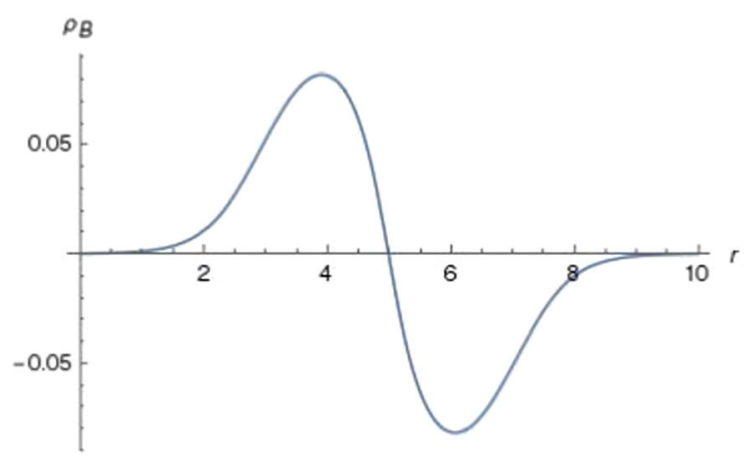

(b) $\rho_{B}(r)$

Fig. 2 Solution with $m=1$ 


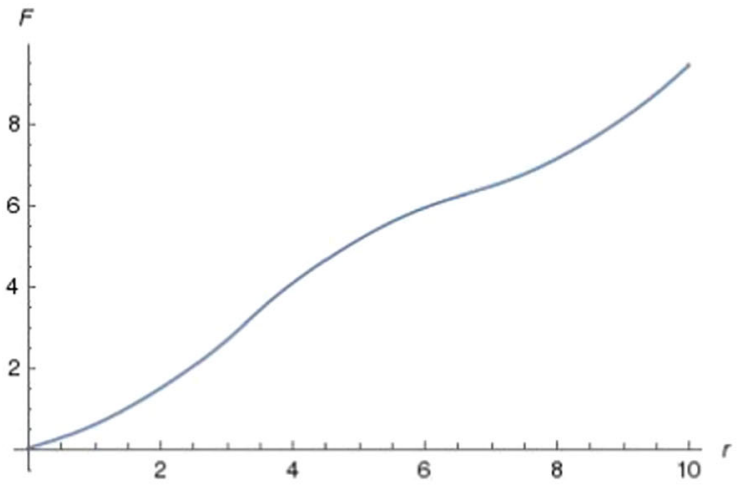

(a) $F(r)$

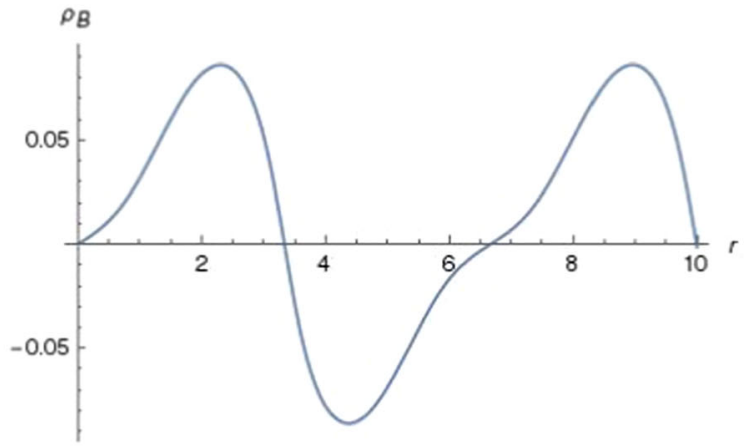

(b) $\rho_{B}(r)$

Fig. 3 Solution with $n=1$

of "bumps" in the charge density is respectively $(2 n+1)$ and $(2 m)$ for configurations of the type (23) and (24). The Fig. 2, in which we adopted periodic boundary conditions (24), represents the cell of a crystal. The building block of such a crystal has two bumps of charges $p$ and $-p$ so that, when it is extended on the plane $\mathbb{R}^{2}$, the final result is an infinite sequence of baryon-antibaryon. Precisely, cutting and gluing the cylinders to form an infinite plane, the crystal solution consists of an infinite sequence of ordered stripes of baryon and anti-baryon matter. This configuration is a 2dimensional example of ordered structure in baryon matter at finite density. Differently, the solutions in Figs. 1 and 3 show respectively one and three bumps of baby baryon matter placed in a finite box of dimension $R \times 2 \pi L$.

All in all, the coupled baby Skyrme field equations Eqs. (19) and (20) with the ansatz in Eq. (16) reduce to a simple quadrature. The boundary condition in Eq. (23), focusing on the case with non-zero charge $B=p$, are

$F(R)=\pi+2 n \pi, F(0)=0, n \in \mathbb{N}$

and thus reduces to:

$I\left(n, p, E_{0}, K, k ; c_{j}\right)=R, \quad E_{0}>0$,

where we define

$$
\begin{aligned}
I\left(n, p, E_{0}, K, k ; c_{j}\right) & \equiv \int_{0}^{\pi+2 n \pi} \frac{d F}{\eta\left(F, E_{0}\right)} \\
& =\int_{0}^{\pi+2 n \pi} \frac{\left(1-K c_{3} \sin ^{2}(F)\right)^{1 / 2}}{\left[2\left(E_{0}-W(F)\right)\right]^{1 / 2}} d F .
\end{aligned}
$$

The above equation for $E_{0}$ always has a positive real solution. $^{2}$ Moreover, one can see that $\partial_{r} F>0$ and that, when $n$ is large, both $\eta\left(F, E_{0}\right)$ and $E_{0}$ are of order $n$.

\footnotetext{
2 The left hand side of Eq. (38) as function of $E_{0}$ increases from very small values (when $E_{0}$ is very large and positive) to very large values (when $E_{0}$ is close to zero but positive). Thus, there is always a value of $E_{0}$ which satisfies Eq. (38).
}

The above integral $I\left(n, p, E_{0}, K, k ; c_{j}\right)$ belongs to the family of (generalized) elliptic integrals at least for integer values of $k$ in the potential (7). Let us consider first the above integral in Eq. (19) (which plays a very important role, as explained in a moment) when $k=1$ and $k=2$. In both cases, $I\left(n, p, E_{0}, K, k ; c_{j}\right)$ in Eq. (39) has the form

$I=\int_{0}^{\pi+2 n \pi} \frac{\left(N_{0}+N_{2} \sin ^{2}\left(\frac{F}{2}\right)+N_{4} \sin ^{4}\left(\frac{F}{2}\right)\right)^{1 / 2}}{\left(D_{0}+D_{1} \sin \left(\frac{F}{2}\right)+D_{2} \sin ^{2}\left(\frac{F}{2}\right)+D_{4} \sin ^{4}\left(\frac{F}{2}\right)\right)^{1 / 2}} d F$,

where the real coefficients $N_{i}$ and can be related with $K, c_{j}$ and $E_{0}$ of the theory. Then, both polynomial of fourth order in $\sin (F / 2)$ can be factorized in their four roots $\left(\delta_{j}\right.$ for the denominator and $v_{j}$ for the numerator, some of them can be complex conjugated):

$I=\int_{0}^{\pi+2 n \pi} \frac{\prod_{i=1}^{4}\left(\sin \left(\frac{F}{2}\right)-v_{i}\right)^{1 / 2}}{\prod_{j=1}^{4}\left(\sin \left(\frac{F}{2}\right)-\delta_{j}\right)^{1 / 2}} d F$.

These types of integral are called "generalized elliptic integrals" in the literature (see $[123,124]$ and references therein). They satisfy recursion relations and their asymptotic expansions are known explicitly. For simplicity we do not include these solutions explicitly here, the interested reader can simply solve equation (28) directly using the DSolve function in Mathematica. On the other hand, when $k$ is too large, it is not possible in general to find explicitly the roots of the polynomial $E_{0}-W(F)$ appearing in the denominator of Eqs. (39) and (40). Thus, in these cases, the theory of generalized elliptic integrals will not help to reduce the integral $I\left(n, p, E_{0}, K, k ; c_{j}\right)$ in Eq. (39) to elementary functions.

Equations (38) and (39) (which implements the boundary condition in order to have topological charge $p$ ) can be seen as a relation between the height of the cylinder $R$, the integration constant $E_{0}$, the topological charge $p$, the kink 
number $2 n+1$ and the coupling constants of the theory. In other words, Eq. (38) represents a sort of equation of state for the system which must be satisfied in order for the solutions to exist.

The conclusion is that the ansatz in Eq. (16) in the flat cylindrical metric in Eqs. (8) and (9) in which the profile $F$ is given in closed form in Eqs. (29), (30) and (38) gives rise to exact and topologically non-trivial solutions (with baby baryonic charge $p$ ) of the field equations for any integers $n, m$ and $p$.

\subsection{Energy density}

From the energy-momentum tensor

$$
\begin{aligned}
T_{\mu \nu}= & -a_{1}\left(\nabla_{\mu} F \nabla_{\nu} F+\sin ^{2} F\left(\nabla_{\mu} G\right)\left(\nabla_{\nu} G\right)\right) \\
& +a_{2}\left\{\operatorname { s i n } ^ { 2 } F \left[\left(\nabla_{\mu} F \nabla_{\nu} F\right)\left(\nabla_{\sigma} G \nabla^{\sigma} G\right)\right.\right. \\
& +\left(\nabla_{\sigma} F \nabla^{\sigma} F\right)\left(\nabla_{\mu} G \nabla_{\nu} G\right) \\
& \left.\left.-2\left(\nabla_{\mu} F \nabla_{\nu} G\right)\left(\nabla_{\sigma} F \nabla^{\sigma} G\right)\right]\right\} \\
& +g_{\mu \nu}\left\{\frac{a_{1}}{2}\left[\nabla_{\sigma} F \nabla^{\sigma} F+\sin ^{2} F\left(\nabla_{\sigma} G \nabla^{\sigma} G\right)\right]\right. \\
& -\frac{a_{2}}{2}\left[\operatorname { s i n } ^ { 2 } F \left(\left(\nabla_{\sigma} F \nabla^{\sigma} F\right)\left(\nabla_{\sigma} G \nabla^{\sigma} G\right)\right.\right. \\
& \left.\left.\left.-\left(\nabla_{\sigma} F \nabla^{\sigma} G\right)^{2}\right)\right]-V\right\}
\end{aligned}
$$

the energy density $T_{00}$ with the ansatz in Eq. (16) reads

$$
T_{00}=\rho=H_{1}(F) \frac{\left(F^{\prime}\right)^{2}}{2}+H_{2}(F),
$$

where

$$
\begin{aligned}
& H_{1}(F)=-a_{1}\left(1-K c_{3} \sin ^{2}(F)\right), \\
& H_{2}(F)=-a_{1} \frac{K}{2} \sin ^{2}(F)+V .
\end{aligned}
$$

We show in Fig. 4 the plots of the energy density for different solutions with the same parameters used in (34).

It is worth to note that since, in this section, we are considering static solutions, the field equations for $F$ in Eq. (26) can also be obtained considering the energy-density as a one- dimensional effective Lagrangian for the static profile $F(r)$. Moreover, (replacing $\left(\partial_{r} F\right)^{2}$ with $\eta\left(F, E_{0}\right)^{2}$ using Eq. (30)), one can get an expression of the energy-density which does not depend on $F^{\prime}$ :

$\rho_{0}(F)=H_{1}(F) \frac{\eta\left(F, E_{0}\right)^{2}}{2}+H_{2}(F)$,

where the integration constant is fixed by equation (38). The plots of the energy density as a function of $r$ from 0 to $R$ reveals $2 n+1$ or $2 m$ bumps. Thus, this family of solutions with charge $p$ is dressed with $2 n+1$ or $2 m$ kinks.

Note also that in this case the energy density of a given field $F$ depending only on one coordinates ( $r$ in our cases) can be written as

$$
\begin{aligned}
T_{00} & =H_{1}(F) \frac{\left(F^{\prime}\right)^{2}}{2}+H_{2}(F) \\
& =H_{1}(F)\left[\frac{\left(F^{\prime}\right)^{2}}{2}+\frac{H_{2}(F)}{H_{1}(F)}\right]
\end{aligned}
$$

(where $H_{j}(F)$ are functions of the field $F$ ) and therefore BPS-like tricks are available. Indeed,

$$
\begin{gathered}
T_{00}=H_{1}(F)\left[\frac{F^{\prime}}{\sqrt{2}} \pm\left(\frac{H_{2}(F)}{H_{1}(F)}\right)^{1 / 2}\right]^{2} \\
\mp \frac{2}{\sqrt{2}}\left(H_{1}(F) H_{2}(F)\right)^{1 / 2} F^{\prime}
\end{gathered}
$$

so that one can "complete the squares" in BPS style, the last term in Eq. (47) being a total derivative. The integral of this total derivative defines a topological charge $Q(n)$

$Q(n)=\int_{0}^{R} \int_{0}^{2 \pi} \frac{2}{\sqrt{2}}\left(H_{1}(F) H_{2}(F)\right)^{1 / 2} F^{\prime} d r L d \phi$

which depends on $n$ (or $m$ ), i.e. the integer which determines the number of bumps defined above). It is obvious that the above expression defines a boundary term: to see this, it is enough to define a function of $F$ (let us denote it by $\Omega(F)$ ) with the following property

$$
\frac{\partial}{\partial F} \Omega(F)=\frac{4 \pi L}{\sqrt{2}}\left(H_{1}(F) H_{2}(F)\right)^{1 / 2},
$$

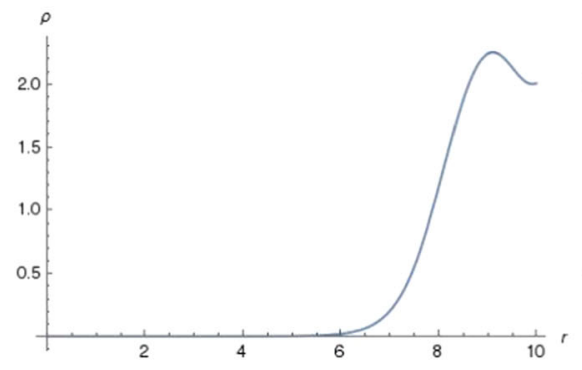

(a) $n=0$

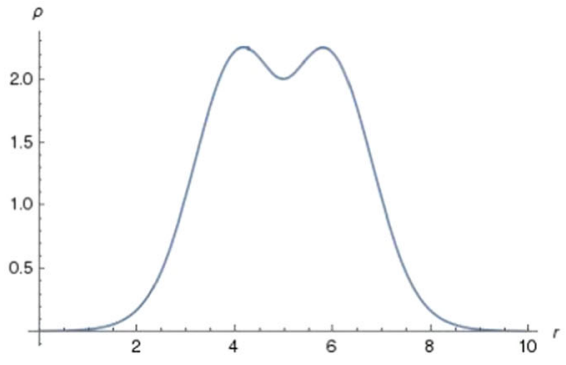

(b) $m=1$

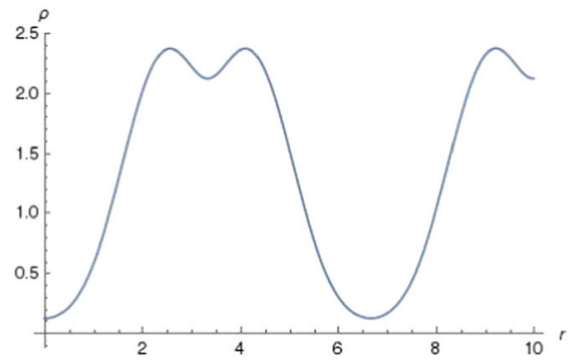

(c) $n=1$

Fig. 4 Energy density 
such a function always exists. Therefore, one gets:

$Q(n)=\Omega(R)-\Omega(0)$,

hence, such new "topological charge" is sensitive to the integer $n$ (or $m$ ) appearing in the boundary conditions. From the physical point of view, this integer $n$ (or $m$ ) measures the number of bumps (local maxima) in the energy density in the $r$ direction. The corresponding BPS bound is

$E \geq|Q(n)|$

It is worth to emphasize that, for the reasons explained above, this "topological charge" is different from the usual winding number of baby Skyrmions. One can see that there exist configurations which saturate the bound: such configurations satisfy

$\frac{F^{\prime}}{\sqrt{2}}= \pm\left(\frac{H_{2}(F)}{H_{1}(F)}\right)^{1 / 2}= \pm\left(\frac{\frac{K}{2} \sin ^{2}(F)+\frac{V}{a_{1}}}{1-K c_{3} \sin ^{2}(F)}\right)^{1 / 2}$

Note that this equation is the same of (28) in the special case of $E_{0}=0$. Remarkably enough, also in this case, the saturation of the above BPS bound does imply the general field equations (compare Eq. (50) with Eqs. (28) and (30)). Thus, the present analytic solutions have two topological labels: the baby baryonic charge $p$ and $Q(n)$ (or simply $n$ or $m$ ). Obviously, the configurations which saturate the above bound are stable. This happens when the integration constant $E_{0}$ in Eqs. (28) and (30) vanishes (which is the case when there is only one bumps in the energy density along the $r$ direction). However, as long as the integration constant $E_{0}$ is such that $F^{\prime}$ does not change sign (see below), also the solutions with higher $n$ are stable (at least under $r$-dependent perturbations of the profile $F$ ).

As a last remark, the total energy for this solutions can also be written in closed form:

$E_{\mathrm{tot}}=2 \pi L \int_{0}^{R} d r T_{00}$.

Due to the fact that the field equation for $F$ is integrable, one can compute explicitly the above integral over $r$. From Eqs. (28) and (30)

$\frac{d F}{\eta\left(F, E_{0}\right)}=d r$

so that taking into account Eqs. (44), (44) and (45) (as well as the boundary conditions on $F$ ) we get

$E_{\mathrm{tot}}=2 \pi L \int_{0}^{F(R)} d F\left[H_{1}(F) \frac{\eta\left(F, E_{0}\right)}{2}+\frac{H_{2}(F)}{\eta\left(F, E_{0}\right)}\right]$.

Also the above explicit integral (which describes how the total energy depends on both topological charges, on the size of the cylinder as well as on the other parameter of the model) belongs to the family of generalized elliptic integrals described above (see [123, 124] and references therein). However, if the $k$ in the potential term is too big, the roots of the polynomial cannot be computed explicitly. On the other hand, the above formula is very useful since it allows to compute explicitly the derivatives of the total energy with respect to relevant parameters.

\subsection{A remark on the stability}

In this section we will look more carefully at the stability of our solutions.

A complete treatment of the stability requires the study of the most generic fluctuations around a background static solution. Using $F$ and $G$ as variables of a vector $\Psi$, we expand the fields as

$\Psi \equiv\left(\begin{array}{c}F(r, \phi) \\ G(r, \phi)\end{array}\right)=\left(\begin{array}{c}F_{0}(r)+\delta F(r, \phi) \\ G_{0}(\phi)+\delta G(r, \phi)\end{array}\right) \equiv \Psi_{0}+\delta \Psi$,

where $F_{0}$ and $G_{0}$ are solutions of the equations of motion and $\delta F$ and $\delta G$ are generic perturbations. Expanding the static energy in term of the perturbation $\delta \Psi$, we arrive at

$E(\Psi)=E_{0}\left(\Psi_{0}\right)+\frac{1}{2} \int d^{2} x \delta \Psi^{\dagger} \widehat{O} \delta \Psi+\cdots$,

where $E_{0}\left(\Psi_{0}\right)$ represents the energy of the baby Skyrmion and the ellipses indicate terms with higher powers of $\Psi$ that can be neglected. The operator $\widehat{O}$ is Hermitian and consists of a $2 \times 2$ matrix whose components are

$\widehat{O} \equiv \widehat{O}_{i j}$

with

$$
\begin{aligned}
\widehat{O}_{11}= & {\left[a_{1}-a_{2} K \sin ^{2}\left(F_{0}\right)\right] \partial_{r}^{2}-a_{2} K \sin \left(2 F_{0}\right) F_{0}^{\prime} \partial_{r} } \\
& -\left[a_{1} K \cos \left(2 F_{0}\right)+a_{2} K \cos \left(2 F_{0}\right)\left(F_{0}^{\prime 2}\right.\right. \\
& \left.+a_{2} K \sin \left(2 F_{0}\right) F_{0}^{\prime \prime}-\frac{a_{0}}{2} \cos \left(F_{0}\right)\right]+\frac{a_{1}}{L^{2}} \partial_{\phi}^{2}
\end{aligned}
$$

$\widehat{O}_{12}=-\frac{a_{1}}{L^{2}} \sin \left(2 F_{0}\right)\left(\partial_{\phi} G_{0}\right) \partial_{\phi}-\frac{a_{2}}{L^{2}} \sin \left(2 F_{0}\right)\left(F_{0}^{\prime 2}\left(\partial_{\phi} G_{0}\right) \partial_{\phi}\right.$

$$
\begin{aligned}
& -\frac{2 a_{2}}{L^{2}} \sin ^{2}\left(F_{0}\right) F_{0}^{\prime \prime}\left(\partial_{\phi} G_{0}\right) \partial_{\phi} \\
& -\frac{a_{2}}{L^{2}} \sin ^{2}\left(F_{0}\right) F_{0}^{\prime}\left(\partial_{\phi} G_{0}\right) \partial_{r} \partial_{\phi} \\
\widehat{O}_{21}= & \frac{a_{1}}{L^{2}} \sin \left(2 F_{0}\right)\left(\partial_{\phi} G_{0}\right) \partial_{\phi}+\frac{a_{2}}{L^{2}} \sin ^{2}\left(F_{0}\right) F_{0}^{\prime \prime}\left(\partial_{\phi} G_{0}\right) \partial_{\phi} \\
& -\frac{a_{2}}{L^{2}} \sin ^{2}\left(F_{0}\right) F_{0}^{\prime}\left(\partial_{\phi} G_{0}\right) \partial_{r} \partial_{\phi} \\
\widehat{O}_{22}= & a_{1} \sin ^{2}\left(F_{0}\right) \partial_{r}^{2}+a_{1} \sin \left(2 F_{0}\right) F_{0}^{\prime} \partial_{r} \\
& -\frac{a_{2}}{L^{2}} \sin ^{2}\left(F_{0}\right)\left(F_{0}^{\prime 2} \partial_{\phi}^{2}+\frac{a_{1}}{L^{2}} \sin ^{2}\left(F_{0}\right) \partial_{\phi}^{2}\right.
\end{aligned}
$$


This operator refers to the case of $k=1$ for the potential (7). To simplify the expression (52), the fluctuation $\delta \Psi$ can be properly decomposed as

$\delta \Psi=\sum_{n} c_{n} \delta \Psi_{n}$,

where the set of functions $\left\{\delta \Psi_{n}\right\}$ is a complete and orthonormal basis representing the set of the eigenfunctions of the operator $\widehat{O}$

$\widehat{O} \delta \Psi_{n}=\lambda_{n} \delta \Psi_{n}$,

with $\left\{\lambda_{n}\right\}$ eigenvalues. Using the property of the basis $\left\{\delta \Psi_{n}\right\}$, the energy (52) reduces to

$E(\Psi)=E_{0}\left(\Psi_{0}\right)+\sum_{n} \frac{\lambda_{n}}{2}\left|c_{n}\right|^{2}+\cdots$

for a whatever perturbation $\delta \Psi$, identified uniquely by the set of coefficients $\left\{c_{n}\right\}$. The stationary point $\Psi_{0}$ in the space of the field configurations is a local minimum of the energy, i.e. a stable field configuration, if and only if $\lambda_{n} \geq 0 \forall n$. Therefore, the problem of the stability, at least under small perturbations, reduces to a problem of eigenvalues for the operator $\widehat{O}$.

In order to resolve the equation for the eigenvalues, we need to specify the boundary condition for the perturbation $\delta F$ and $\delta G$. These conditions must be chosen in such a way to not change the total topological charge (9) and, moreover, to not change the number of "bumps", i.e. the number of baryons and anti-baryons, on the cylinder. In particular, for background solutions of the type (23) we request periodic boundary conditions for the fluctuations

$$
\begin{aligned}
& \delta F(r, 0)=\delta F(r, 2 \pi), \\
& \delta G(r, 0)=\delta G(r, 2 \pi), \\
& \delta F(0, \phi)=\delta F(R, \phi), \\
& \delta G(0, \phi)=\delta G(R, \phi) .
\end{aligned}
$$

The periodicity along the $\theta$-coordinate is due to the cylindrical geometry. Otherwise, the condition along $r$ depends on the periodicity of the background configuration due to (24). In the case of background with condition (23), we need instead

$$
\begin{aligned}
& \delta F(r, 0)=\delta F(r, 2 \pi), \\
& \delta G(r, 0)=\delta G(r, 2 \pi), \\
& \delta F(0, \phi)=\delta F(R, \phi)=0 .
\end{aligned}
$$

Although numerical calculations are needed for the calculation of the eigenvalues $\left\{\lambda_{n}\right\}$, analytically we are able to calculate the zero modes $\left(\lambda_{n}=0\right)$ of the operator (53). One of these zero modes arises from the $S O(2)$ exact symmetry of the Lagrangian and can be written explicitly as $\delta F(r, \phi)=0$

$\delta G(r, \phi)=\alpha$

where $\alpha$ is a constant angle. This solution respects both the boundary conditions independently from the background field. Otherwise, the periodicity of the background solution $F_{0}$ built with (23) gives rise to a translational zero mode

$F_{0}(r) \rightarrow F_{0}(r+\epsilon)$,

and then to the eigenvector

$\delta F(r, \phi)=\partial_{r} F_{0}(r)$,

$\delta G(r, \phi)=0$,

respecting the conditions (61). This last eigenvector solution belongs to that kind of fluctuations of the form

$\delta F=\delta F(r)$,

$\delta G=0$,

i.e. fluctuations involving only the field $F$ with the propriety of keeping the structure of the ansatz (16). Reducing the space of perturbations only to the perturbations of the type (66), the eigenvalues system of equations (59) reduces to a 1dimensional equation. Using the oscillation/nodal theorem, we identify the eigenfunction (65) with the groundstate of the system being such a solution with no node. Since in this case the groundstate has $\lambda_{0}=0$, then $\lambda_{n} \geq 0$. As a remark, we can affirm that the baby Skyrmion solutions with boundary (23) are stable under any fluctuation of the type (66).

As anticipated before, beyond the analytic calculations, the set of eigenvalues for a generic fluctuation that respects the boundary conditions (61) or (62) has been calculated numerically. When possible, we used the Mathematica builtin function NDEigensystem and when this failed we proceeded by discretizing the coordinate space of the operator manually.

This discretization is achieved by using a uniform 2dimensional lattice of size $R \times 2 \pi L$ in the $\theta$ and $r$ directions. We vary the number of points in order to check the convergence of our procedure. The derivatives are discretized using second-order finite differences, adapted to backwards and forward differences in the $r$ boundaries, and periodic conditions on the $\theta$ ones. This generates a large matrix of the discretised operator, which is then passed through the Eigenvalues function in Mathematica, to extract the required eigenvalues.

In this paper, we examine the stability of the static solutions characterized by $n=0, m=1, n=1$ in (23), (24) with parameters (34). The study of the small perturbations around the solution $m=1$ has been realized with the use of NDEigensystem, calculating the smallest 400 eigenvalues 
in order of absolute value for the operator (53). Our analysis reveals no negative eigenvalues among this set of values. In the case of solutions $n=0$ and $n=1$, the more complicated boundary conditions (62) need the implementation of the eigenvalue problem on a lattice. For these solutions, the numerical procedure reveals no negative eigenvalues up to numerical accuracy $\mathcal{O}\left(10^{-3}\right)$. We therefore conclude that these solutions are stable.

As a remark, since the topological charge does not depend on $m$ or $n$, all the solutions with $m>0$ or $n>0$ have to be considered metastable. In other words, in every topological sector the configurations with $m>0$ or $n>0$ represent local minima of the energy. The stability (or meta-stability) of a sequence of baby Skyrmions and anti-baby skyrmions represent a rather surprising result of this work. From one side, it constitutes an effect of the finite density approach since the system is forced to be confined in a finite box (a finite cylinder in this case). Moreover, the baby skyrmions built on a cylinder with the ansatz (16) seem to have different features compared with the solutions built on the $\mathbb{R}^{2}$ plane. For example, on the plane a baby and an anti-baby skyrmion can be distinguished from the different orientation of the map $G$ in (3) with respect to the polar angle $\theta$. Otherwise, in the ansatz (16) the map $G$ does not change and the sign of the topological density depends on the orientation of the map $F$ on the sphere. In general, the different geometry between the plane and the cylinder leads to different characteristics for the solitons. As a consequence, we expect a different kind of interaction among them. The analysis of these new interactions can be an interesting point for a future work.

3.4 The case of potential $V=\frac{1}{2}\left(1-\phi_{3}^{2}\right)$

In this section, we consider the baby Skyrmion theory defined on a cylinder with potential

$V=a_{0}\left(\frac{1-\phi_{3}^{2}}{2}\right)$

instead of $V=\frac{a_{0}}{2}\left(1-\phi_{3}\right)$ in (7). Using again the ansatz (16), the equations of motion with the new potential are the same as Eq. (19) and (20) replacing $V$ with the expression (67). All the considerations discussed before about the topology of these solutions hold and again the baby Skyrmions are classified by the topological charge $B=p$ and by the integers $n$ and $m$. In the following, we show the plots of some solutions calculated with this set of parameters

$$
\begin{array}{lll}
a_{0}=1 & L=1 & p=1 \\
a_{1}=-1 & R=10 & K=1 . \\
a_{2}=1 & &
\end{array}
$$

Using equation (28) for the profile function $F(r)$ and the set of external parameters (68), the constants $E_{0}$ for the solutions $n=0, m=1, n=1$ are respectively

$$
\begin{array}{ll}
n=0 & E_{0}=0 \\
m=1 & E_{0}=0.0305 \\
n=1 & E_{0}=0.256 .
\end{array}
$$

The plots obtained with Eqs. (19) or (28) are totally equivalent.

The study of the stability follows the same analysis of Sect. 3.3 in which the operator $\widehat{O}_{i j}$ has the same expression of (53) replacing $\widehat{O}_{11}$ with

$$
\begin{aligned}
\widehat{O}_{11}= & {\left[a_{1}-a_{2} K \sin ^{2}\left(F_{0}\right)\right] \partial_{r}^{2}-a_{2} K \sin \left(2 F_{0}\right) F_{0}^{\prime} \partial_{r} } \\
& -\left[a_{1} K \cos \left(2 F_{0}\right)+a_{2} K \cos \left(2 F_{0}\right)\left(F_{0}^{\prime 2}\right.\right. \\
& \left.+a_{2} K \sin \left(2 F_{0}\right) F_{0}^{\prime \prime}-a_{0}\left(\cos ^{2}\left(F_{0}\right)-\sin ^{2}\left(F_{0}\right)\right)\right] \\
& +\frac{a_{1}}{L^{2}} \partial_{\phi}^{2} .
\end{aligned}
$$

The procedure and the conclusions of our analysis about the eigenvalues of this new operator are equivalent to the case of Sect. 3.3. About the solution $m=1$, using NDEigensystem, we do not find any negative eigenvalues among the smallest 400 eigenvalues in order of absolute value for the operator (53) with (72). In the case of solutions $n=0$ and $n=1$, the numerical computation on the lattice once again reveals the presence of no negative values up to numerical error.

In the case of the two-vacuum potential is possible to build the baby skyrmion on an infinite cylinder keeping the energy finite. To this end, in the simple $n=0$ configuration, we must impose the boundary conditions for $F$ as

$F(-\infty)=0, \quad F(\infty)=\pi$.

This solution has been discussed in [126] with the name of "Skyrmion wall". A further analysis of these kind of solutions and their stability can be extended to the case of $n>0$ or $m>0$ in a future work.

\section{Gauged baby Skyrmions}

We are finally ready to consider the system coupled to the gauge fields. Despite its high interest, very few analytic solutions have been found in this system. In this subsection we will consider the gauged baby Skyrme action in Eq. (1) and we set the coupling $e=1$. This action is invariant under the $S O(2) \simeq U(1)$ gauge transformation

$$
\begin{aligned}
& F \rightarrow F^{\prime}=F \\
& G \rightarrow G^{\prime}=G+\alpha(x) \\
& A_{\mu} \rightarrow A_{\mu}^{\prime}=A_{\mu}-\partial_{\mu} \alpha(x) .
\end{aligned}
$$

The Maxwell equations corresponding to the above action read

$$
-\nabla^{\mu} F_{\mu \nu}=\frac{a_{1}}{2} \sin ^{2}(F) \frac{\delta}{\delta A^{\nu}}\left[\left(\nabla_{\mu} G+A_{\mu}\right)\left(\nabla^{\mu} G+A^{\mu}\right)\right]
$$




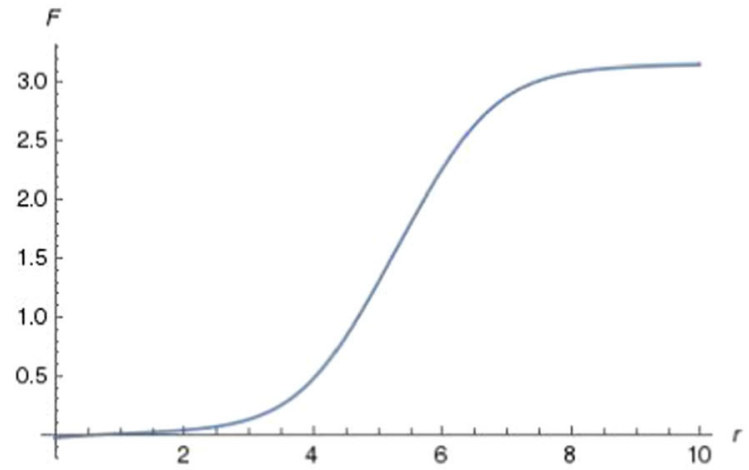

(a) $F(r)$

Fig. 5 Solution with $n=0$

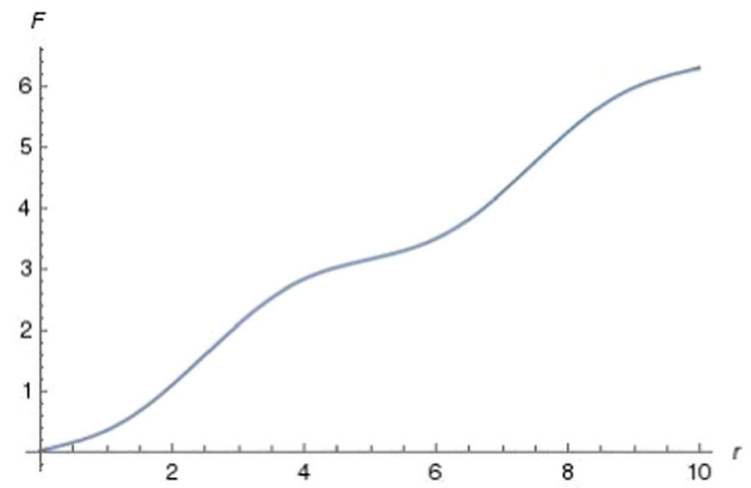

(a) $F(r)$

Fig. 6 Solution with $m=1$

$$
\begin{aligned}
& -\frac{a_{2}}{2} \sin ^{2}(F) \frac{\delta}{\delta A^{\nu}}\left[\left(\nabla_{\mu} F\right)\left(\nabla^{\mu} F\right)\left(\nabla_{\nu} G+A_{\nu}\right)\left(\nabla^{\nu} G+A^{\nu}\right)\right. \\
& \left.-\left(\left(\nabla_{\mu} F\right)\left(\nabla^{\mu} G+A^{\mu}\right)\right)^{2}\right] .
\end{aligned}
$$

As it will be explained in the next subsection here below, the approach of [84-92] leads to the ansatz (3) with

$F=F(r), G=p \phi-\omega t$, with $\omega=\frac{p}{L}$

so that

$\nabla_{\mu} F \nabla^{\mu} G=\nabla_{\mu} G \nabla^{\mu} G=0$

and for the gauge field

$A_{\mu}=(-\eta(r), 0, L \eta(r))$.

4.1 On the construction of the ansatz for the gauged solitons

Here we will explain the reasoning behind the ansatz defined here above for the gauged baby-Skyrmion as well as for the corresponding gauge potential. Generically, if one replaces (in the field equations of the gauged baby Skyrme model) the derivative with the $U(1)$ covariant derivative (as defined in

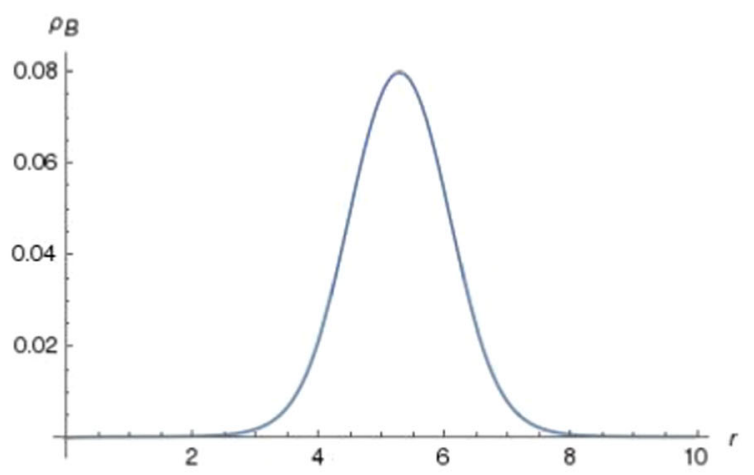

(b) $\rho_{B}(r)$

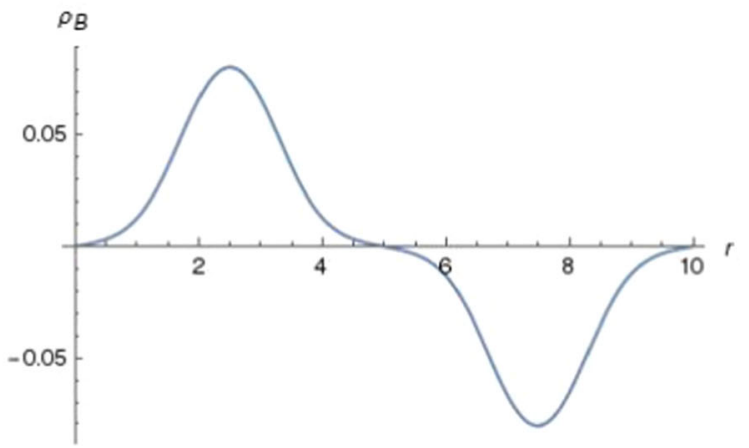

(b) $\rho_{B}(r)$

Eq. (2)), then one gets many new interactions terms coupling the baby Skyrmion with $A_{\mu}$.

Therefore, a relevant question is:

Is it possible to find an ansatz for $A_{\mu}$ and for the baby Skyrmion able to keep as much as possible the very nice properties of the ansatz in Eq. (16) which lead the analytic solutions described in the previous sections in the ungauged case (keeping alive the topological charge)?

In order to achieve this goal, it is enough to demand

$$
\begin{aligned}
& \nabla^{\mu} A_{\mu}=0, A_{\mu} A^{\mu}=0, \\
& A_{\mu} \nabla^{\mu} F=0, A_{\mu} \nabla^{\mu} G=0, \\
& \nabla^{\mu} F \nabla_{\mu} G=0, \nabla_{\mu} G \nabla^{\mu} G=0,
\end{aligned}
$$

This strategy has been inspired by the similar (but more involved) case of the gauged Skyrme model in $(3+1)$ dimensions: see [85,89-91]. The above conditions in Eqs. (79) and (80) require that $A_{\mu}$ must have the following form:

$A_{\mu}=(-\eta(r), 0, L \eta(r))$,

which has been introduced in Eq. (78). The reasoning behind the conditions in Eqs. (79) and (80) is the following. All the 


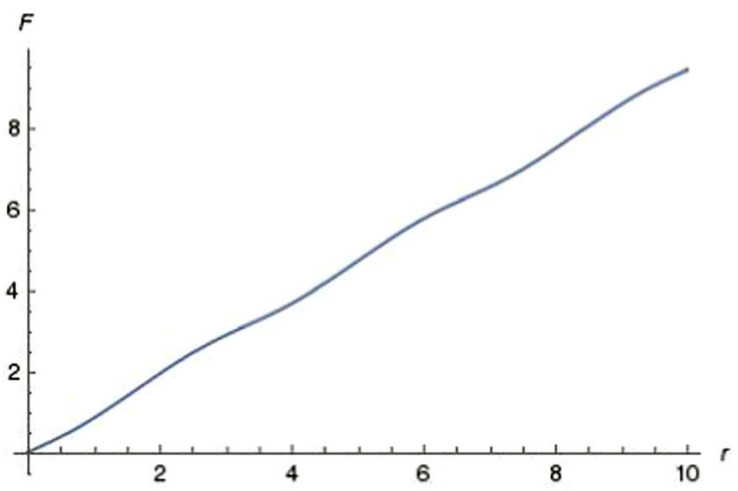

(a) $F(r)$

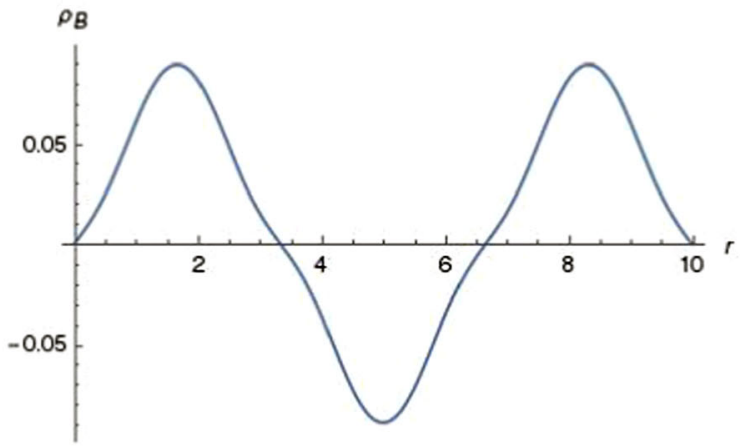

(b) $\rho_{B}(r)$

Fig. 7 Solution with $n=1$

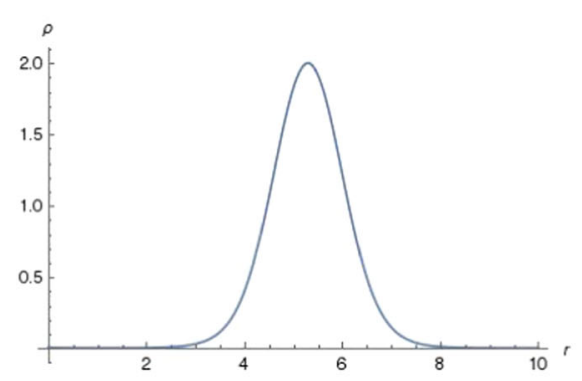

(a) $n=0$

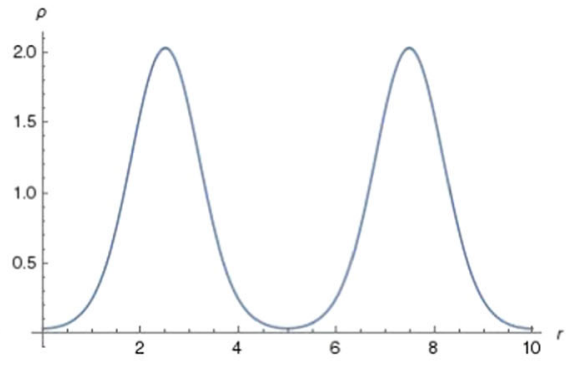

(b) $m=1$

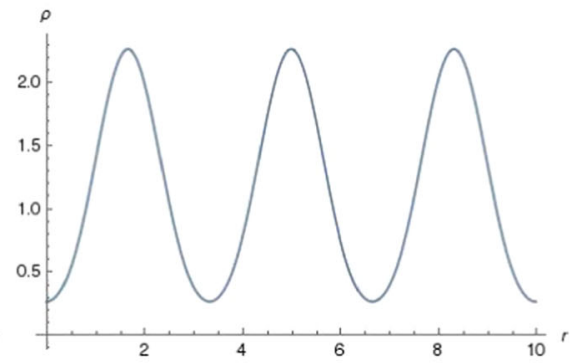

(c) $n=1$

Fig. 8 Energy density

terms in the field equations for the gauged baby Skyrmions which couple the gauge potential $A_{\mu}$ with the degrees of freedom $F$ and $G$ of the baby Skyrmion involve (at least) one of the following four quantities $X_{j}(j=1,2,3,4)$ :

$$
\begin{aligned}
& X_{1}=\nabla^{\mu} A_{\mu}, X_{2}=A_{\mu} A^{\mu}, \\
& X_{3}=A_{\mu} \nabla^{\mu} F, X_{4}=A_{\mu} \nabla^{\mu} G .
\end{aligned}
$$

Consequently, if one requires that $X_{j}=0$ for $j=1,2,3,4$ then all the terms with $A_{\mu}$ in the field equation of the gauged baby Skyrmion disappear so that the field equations of the gauged baby Skyrme model reduce to the field equations of the ungauged case (analyzed and solved in the previous sections). Of course, one may wonder whether or not the requirement $X_{j}=0$ for $j=1,2,3,4$ can be satisfied by some non-trivial gauge potential, but one can check directly that the $A_{\mu}$ in Eq. (78) does the job. However, Eqs. (79) and (80) are not enough yet to achieved the desired goal. In particular, we remind the reader that we are considering a model in $(2+1)$ dimensions and the degrees of freedom of the baby Skyrmion are encoded in the scalar functions $F$ and $G$ which must be independent (namely $d F \wedge d G \neq 0$ ) in order to have non-vanishing topological charge. Moreover, in order to simplify as much as possible the field equations in the ungauged case without loosing the topological charge we had to require also $\nabla^{\mu} F \nabla_{\mu} G=0$. The problem is that this last condition (if both $F$ and $G$ only depend on spacelike coordinates) could be incompatible with the conditions $X_{3}=0=X_{4}=X_{2}$ (where the $X_{j}$ have been defined in Eq. (83)). Indeed, in this case we would not have enough independent space-like coordinates to satisfy both $\nabla^{\mu} F \nabla_{\mu} G=0$ and $X_{3}=0=X_{4}=X_{2}$ with some non-trivial ${ }^{3} A_{\mu}$. On the other hand, if $G$ depends also on time (as suggested in the discussion in the previous sections on the avoidance of the Derrick theorem) there is a way out. Indeed, if $G$ depends both on $\phi$ and on $t$ in such a way that its gradient is a lightlike vector, then we can satisfy both $\nabla^{\mu} F \nabla_{\mu} G=0$ as well as $X_{3}=0=X_{4}=X_{2}$. The good choice of $G$ is the one in Eq. (76). In this way, all the terms which, in principle, can couple $A_{\mu}$ to $\vec{\Phi}$ in the gauged baby Skyrme field equations vanish and the gauged baby Skyrme field equations reduce, as promised, to the ungauged ones without loosing the topological charge. A further byproduct of the analysis is that also the

\footnotetext{
${ }^{3}$ This is easy to see: when $F$ and $G$ only depend on spacelike coordinates, their gradients only have one non-zero component $\left(\nabla_{r} F\right.$ and $\left.\nabla_{\phi} G\right)$. In this case $A_{\mu}$ cannot be at the same time null (namely $A_{\mu} A^{\mu}=0$ )-as required to simplify the field equations-as well as perpendicular to $\nabla_{\mu} F$ and $\nabla_{\mu} G$. However, if $G$ also depends on time, then $\nabla_{\mu} G$ can be a light-like vector satisfying all the relevant conditions.
} 
Maxwell equations with the $U(1)$ current coming from the gauged baby Skyrme model reduce to one one linear equation as we will now discuss. This is the reasoning behind the present strategy strategy, which has been proven effective not only in this case, but also in the $(3+1)$-dimensions with $S U(N)$ internal symmetry group (see [85,89-91] and references therein).

The components of the tensor $F_{\mu \nu}$ corresponding to the $A_{\mu}$ in Eq. (78) define the electric-like and magnetic-like fields as

$F_{\mu \nu}=\left[\begin{array}{ccc}0 & E_{r} & E_{\phi} L \\ -E_{r} & 0 & -B L \\ -E_{\phi} L & B L & 0\end{array}\right]=\left[\begin{array}{ccc}0 & \partial_{r} \eta & 0 \\ -\partial_{r} \eta & 0 & \partial_{r} \eta L \\ 0 & -\partial_{r} \eta L & 0\end{array}\right]$.

For the reasons we have explained here above, with the above ansatz for the gauge potential $A_{\mu}$ and for the baby Skyrmion, it is possible to reduce the complete set of coupled gauged baby Skyrme model field equation in Eqs. (19), (20) together with the corresponding Maxwell equations in Eq. (75) to a single integrable equation for the profile $F$ as well as to only one linear Schrodinger-like equation in which the effective periodic potential can be computed explicitly in terms of the profile $F$ itself. Obviously, the system is still coupled since the $U$ (1) current corresponding to the gauged baby Skyrmion (which appears on the right hand side of the Maxwell equations) depends explicitly on $\vec{\Phi}$. However, the present approach greatly simplify the coupled field equations of the Maxwell gauged baby Skyrme system since (thanks to Eqs. (79), (80) and (81)) one can first solve the field equations for $\vec{\Phi}$ (in which $A_{\mu}$ disappeared) and then, once $\vec{\Phi}$ is known, one is just left with the Maxwell equations which, in fact, reduce to only one linear equation for $\eta(r)$ in which $\vec{\Phi}$ plays the role of an effective potential. Note that here no approximation will be made: namely we are dealing with the complete set of five coupled non-linear field equations for the gauged baby Skyrme model in (2+1)-dimensions in which both the back-reaction of the solitons on Maxwell field and viceversa are explicitly taken into account in a self-consistent way.

More concretely, the problem to construct analytically gauged baby Skyrmions problem can be divided into two steps.

First step: with the ansatz in Eqs. (76), (78) the gauged baby Skyrme model field equations reduce to a single equation for the profile $F$ (in which neither $A_{\mu}$ nor $a_{2}$ appear explicitly):

$F^{\prime \prime}+\frac{a_{0}}{a_{1}} \frac{\partial}{\partial F}\left(\frac{1-\cos F}{2}\right)^{k}=0$.

Therefore, the above ansatz is able to reduce in a selfconsistent way (without any approximation) the gauged baby Skyrme equations with a non-trivial gauge potential to just one equation for $F$ which is integrable in the sense that it can always be reduced to a quadrature. When $k=1$ or $k=2$, Eq. (85) can be integrated explicitly in terms of inverse elliptic functions (Jacobi Amplitude functions [125], as the reader can quickly verify solving the differential equation below) observing that it is equivalent to the following first order equation

$F^{\prime}= \pm\left[2\left(E_{0}-\frac{a_{0}}{a_{1}}\left(\frac{1-\cos F}{2}\right)^{k}\right)\right]^{1 / 2}$.

The integration constant $E_{0}$ can determined requiring to have the correct boundary conditions (23), (24) in order to obtain a non-vanishing integer topological charge.

Second step: the three Maxwell equations with the current in Eq. (75) corresponding to the gauged soliton reduce consistently to only one equation for $\eta(r)$ (defined in Eq. (78)):

$\frac{\partial^{2} \eta}{\partial r^{2}}+V \eta=\sigma$,

where

$$
\begin{aligned}
& V=a_{1} \sin ^{2}(F)\left[1-\frac{a_{2}}{a_{1}}\left(F^{\prime}\right)^{2}\right], \\
& \sigma=-a_{1} \omega \sin ^{2}(F)\left[1-\frac{a_{2}}{a_{1}}\left(F^{\prime}\right)^{2}\right] .
\end{aligned}
$$

Note that, since the equation for $F$ is solvable (see Eq. (86)), both $\sin ^{2}(F)$ and $\left(F^{\prime}\right)^{2}$ above are known explicitly. It is useful to rewrite Eq. (87) as

$\frac{\partial^{2}}{\partial r^{2}} \Psi+V \Psi=0$,

where

$\Psi=\eta+\omega$.

Here it is also useful to write explicitly the $U(1)$ conserved current $J_{\mu}$ in the general form

$$
\begin{aligned}
J_{\mu}= & -\left[a_{1} \sin ^{2}(F)\left(\nabla_{\mu} G+A_{\mu}\right)\right. \\
& -a_{2} \sin ^{2}(F)\left(\left(\nabla^{v} F \nabla_{\nu} F\right)\left(\nabla_{\mu} G+A_{\mu}\right)\right. \\
& \left.\left.-\left(\nabla^{v} F\left(\nabla_{\nu} G+A_{\nu}\right)\right) \nabla_{\mu} F\right)\right]
\end{aligned}
$$

that due to the ansatz in Eqs. (76), (78) reduces to:

$J_{\mu}=-a_{1} \sin ^{2}(F)\left[1-\frac{a_{2}}{a_{1}}\left(F^{\prime}\right)^{2}\right]\left(\nabla_{\mu} G+A_{\mu}\right)$.

The components of $J_{\mu}$ represent the source of the electric and magnetic field

$J_{\mu}=\left(-\rho_{e}, J_{r}, J_{\phi} L\right)$

where $\rho_{e}$ is the electric charge density and $J_{r}, J_{\phi}$ the currents.

The new energy-momentum tensor with the gauge field $A_{\mu}$ reads

$T_{\mu \nu}=-a_{1}\left(\nabla_{\mu} F \nabla_{\nu} F+\sin ^{2} F\left(\nabla_{\mu} G+A_{\mu}\right)\left(\nabla_{\nu} G+A_{\nu}\right)\right)$ 


$$
\begin{aligned}
& +a_{2}\left\{\operatorname { s i n } ^ { 2 } F \left[\left(\nabla_{\mu} F \nabla_{\nu} F\right)\left(\nabla_{\sigma} G+A_{\sigma}\right)\left(\nabla^{\sigma} G+A^{\sigma}\right)\right.\right. \\
& +\left(\nabla_{\sigma} F \nabla^{\sigma} F\right)\left(\nabla_{\mu} G+A_{\mu}\right)\left(\nabla_{\nu} G+A_{\nu}\right) \\
& \left.\left.-2 \nabla_{\mu} F\left(\nabla_{\nu} G+A_{\nu}\right)\left(\nabla_{\sigma} F\right)\left(\nabla^{\sigma} G+A^{\sigma}\right)\right]\right\}+F_{\mu \sigma} F_{\nu}^{\sigma} \\
& +g_{\mu \nu}\left\{\frac{a_{1}}{2}\left[\nabla_{\sigma} F \nabla^{\sigma} F+\sin ^{2} F\left(\nabla_{\sigma} G+A_{\sigma}\right)\left(\nabla^{\sigma} G+A^{\sigma}\right)\right]\right. \\
& -\frac{a_{2}}{2}\left[\operatorname { s i n } ^ { 2 } F \left(\left(\nabla_{\rho} F \nabla^{\rho} F\right)\left(\nabla_{\sigma} G+A_{\sigma}\right)\left(\nabla^{\sigma} G+A^{\sigma}\right)\right.\right. \\
& \left.\left.\left.-\left(\nabla_{\sigma} F\left(\nabla^{\sigma} G+A^{\sigma}\right)\right)^{2}\right)\right]-V-\frac{1}{4} F_{\sigma \rho} F^{\sigma \rho}\right\}
\end{aligned}
$$

and the energy density $\rho$ with the ansatz (76), (78) reduces to

$$
\begin{aligned}
\rho= & T_{00} \\
= & -a_{1} \sin ^{2}(F)(\omega+\eta)^{2}\left(1-\frac{a_{2}}{a_{1}}\left(F^{\prime}\right)^{2}\right)-a_{1} \frac{\left(F^{\prime}\right)^{2}}{2} \\
& +a_{0}\left(\frac{1-\cos F}{2}\right)^{k}+\left(\eta^{\prime}\right)^{2} .
\end{aligned}
$$

The expression for the topological charge $B$ differs from the ungauged case due to the presence of $A_{\mu}$ as anticipated in (17). The explicit calculation for $B$ with the ansatz (78) gives

$$
\begin{aligned}
B & =\frac{1}{4 \pi} \int d r d \phi\left[\sin F \partial_{r} F\left(\partial_{\phi} G+A_{\phi}\right)+F_{r \phi}(1-\cos F)\right] \\
& =\frac{1}{4 \pi} \int d r d \phi\left[p \sin F \partial_{r} F+L \partial_{r}(\eta(1-\cos F))\right] .(94)
\end{aligned}
$$

As expected, the topological charge depends on the boundary conditions of the fields as (recall we always take $F(0)=0$ as in (23), (24))

$B= \begin{cases}p+L \eta(R) & \text { if } \quad F(R)=(2 n+1) \pi \\ 0 & \text { if } \quad F(R)=2 \pi m .\end{cases}$

In order to keep the topological charge unchanged, the last result imposes one boundary condition for the field $\eta$

$\eta(R)=0$.

This condition is a natural choice since we do not expect that the Maxwell field $A_{\mu}$ introduces a baryon charge in the system. However, mathematically this condition can be relaxed if we simply ask the charge $B$ to be an integer independently of which field "carries" the baryon charge. In this case, the most general condition for $\eta$ becomes

$\eta(R)=\frac{d}{L}, \quad d \in \mathbb{Z}$.

The discussion of this point is beyond the aim of this paper and only the condition (96) will be used.

Now, let us consider the simplest non-trivial example of analytic gauged baby Skyrmion which can be constructed with vanishing potential $a_{0}=0$. In this case, using the ansatz defined in Eqs. (76), (78), one can easily see that the profile $F$ must be linear:

$F(r)=k_{1} r$,

(as in this way Eq. (85) is satisfied) where $k_{1}$ must be chosen in such a way to satisfy the boundary conditions in Eq. (23)

$F(R)=\pi, F(0)=0 \Longrightarrow k_{1}=\frac{\pi}{R}$.

With the above expression for the profile $F(r)$, the remaining Maxwell equation (88) reduces to

$$
\begin{aligned}
{\left[\frac{\partial^{2}}{\partial r^{2}}+a_{1} g \sin ^{2}\left(\frac{\pi}{R} r\right)\right] \Psi } & =0, \\
{\left[1-\frac{a_{2}}{a_{1}}\left(\frac{\pi}{R}\right)^{2}\right] } & =g .
\end{aligned}
$$

In order to solve this equation we need to specify the boundary conditions for $\Psi$. One condition is fixed only for the case of $F$ with boundary (23) by Eq. (96), i.e.

$\Psi(R)=\omega$.

Otherwise, for the condition at $r=0$ there is not a unique choice. The different boundary conditions that we impose to $\Psi$ fix the electric charge and the current inside the system, leading to different physical configurations. In this paper, we show and consider only three cases

$\Psi(0)=\left\{\begin{array}{c}\omega \\ 0 \\ -\omega\end{array}\right.$

but other choices are allowed. In the following, we use for simplicity the same conditions (103), (102) also for the case of $F$ with boundary (24), even if there are not any constraints from the topological charge (95).

We illustrate some representative solutions in Figs. 9, 10 and 11 which are solved with symmetric boundary conditions $\Psi(0)=\Psi(R)=\omega$ with the set of parameters

$\begin{array}{llrl}a_{0} & =0 & & L=1 \\ a_{1} & =-1 & & R=10 \\ a_{2} & =1 & & p=1 .\end{array}$

Figures 9, 10 and 11 show respectively solutions with $n=0$, $m=1$ and $n=1$. For every solution the plots represent the profile $\Psi(r)$, the magnetic field $B(r)$, the electric current $J_{\phi}(r)$ and the energy density $\rho(r)$. In all the cases, the electric field $E_{r}=-B$ as shown in (84), the electric charge density $\rho_{e}=J_{\phi}$ due to (90) and $E_{\phi}=0, J_{r}=0$. As it is clear form the plots, the periodic boundary conditions for $\Psi$ give rise to a positive angular current $J_{\phi}$ which generate a magnetic field with opposite sign on the borders of the cylinder. In the same way, a positive electric charge exists and it 
Fig. 9 Profiles for the gauged baryon solution $n=0$ with periodic boundaries for $\Psi$. The profile function $F(r)$ is defined by $E_{0}=\pi^{2} / 2 R^{2}$

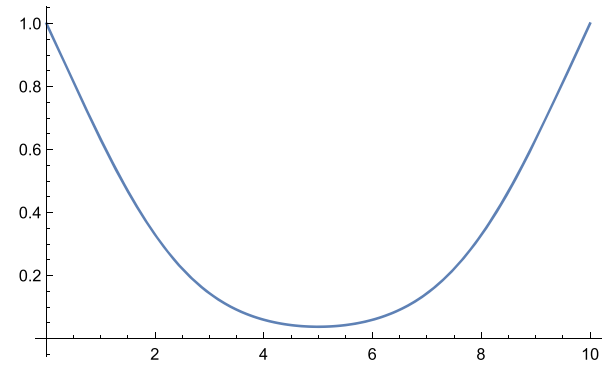

(a) $\Psi(r)$

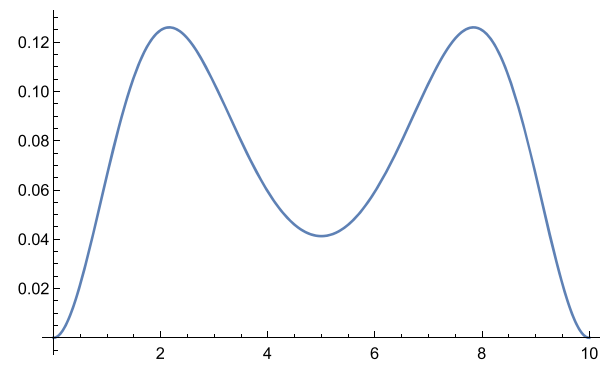

(c) $J_{\phi}(r)$

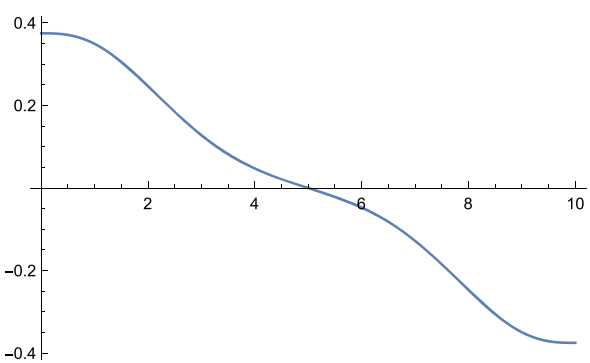

(b) $B(r)$

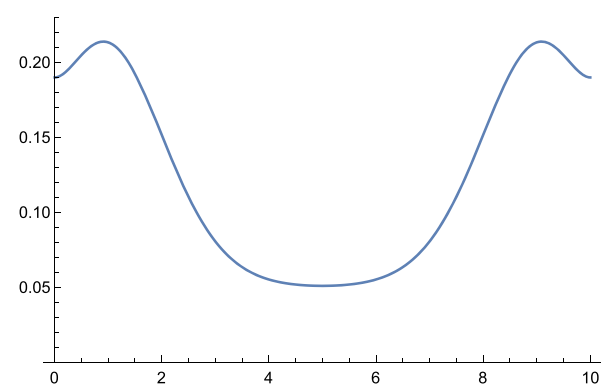

(d) $\rho(r)$ generate an electric field $E_{r}$ that spreads in opposite directions. The energy density $\rho$ is modified by the presence of the electric/magnetic field and it is mainly concentrated at the boundaries of the cylinder.

In Fig. 12, we show the case of anti-periodic boundary conditions for $\Psi$, i.e. $\Psi(0)=-\Psi(R)=-\omega$, only for the solution $n=0$ using again the parameters (104) (the solutions $m=1$ and $n=1$ have a similar profile as it happens for the previous case). This configuration is characterized by a negative current on one half of the cylinder and a positive current, flowing in the opposite $\phi$-direction, on the other half. The magnetic field is therefore negative everywhere. Consistently, the presence of a negative and positive electric charge leads to an electric field $E_{r}$ which goes in the same direction everywhere along the cylinder. The energy density has the identical profile of the periodic case of Fig. 9. The periodic and anti-periodic $\Psi$-configurations are therefore degenerate in energy.

In Fig. 13 we impose a vanishing boundary condition for $\Psi$ at one side of the cylinder, i.e. $\Psi(0)=0$ and $\Psi(R)=\omega$, for the $n=0$ solution with the set of parameters (104). In this case a positive current flows on the cylinder along the $\phi$-direction, similarly to Fig. 9. However, here the magnetic field is unexpectedly only negative. Looking at the electric side, we have a positive electric charge whose electric field goes only to one direction instead of spreading its flux in the two opposite sides.

We can also investigate what happens in the more complicated case of $a_{0} \neq 0$. In particular, we set $a_{0}=1$ for equation (86) and the other parameters are the same of (104).
We also choose $k=1$ for the potential. The solutions are calculated imposing periodic boundary conditions for $\Psi$, i.e. $\Psi(0)=\Psi(R)=\omega$, respectively for $n=0$ and $m=1$ in Figs. 14 and 15. These configurations follow the same considerations that we discussed for Figs. 9 and 10 with the difference, for the case $n=0$, that the potential breaks the symmetry with respect to $R / 2$.

The conclusion of this section is that the coupled system of 5 coupled non-linear field equations made by the 2 field equations of the gauged non-linear baby Skyrme model and the 3 Maxwell equations with the current corresponding to the baby Skyrme model itself (in a sector with non-trivial topological charge) reduce (using the ansatz in Eqs. (76), (78)) exactly to just one linear equation. This equation can be regarded as a Schrodinger-like equation with an effective potential in Eq. (88) which is known explicitly since the baby Skyrme model equations are solved by Eq. (86).

\subsection{Gauged baby Skyrmions and resurgence}

We now discuss an interesting interplay between the present framework and the resurgence scheme. A very difficult problem in the application of resurgence in non-integrable theories with multi-solitonic configurations is the correct identification of the coupling constant which is responsible for the resurgent behavior itself. When finite density effects are present, the combination of parameters of the theory which plays the role of effective coupling constant can be far from obvious. Besides the "obvious" coupling constant (which plays the role of $\hbar$ in the action), other important parameters 
Fig. 10 Profiles for the gauged baryon-antibaryon solution $m=1$ with periodic boundaries for $\Psi$. The profile function $F(r)$ is defined by $E_{0}=2 \pi^{2} / R^{2}$

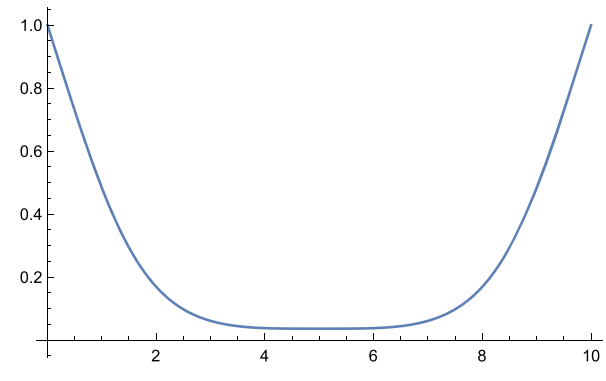

(a) $\Psi(r)$

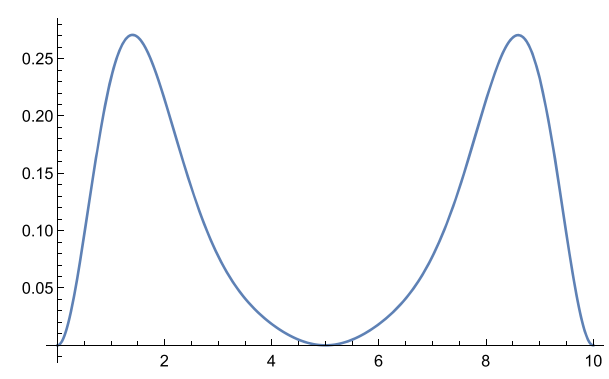

(c) $J_{\phi}(r)$

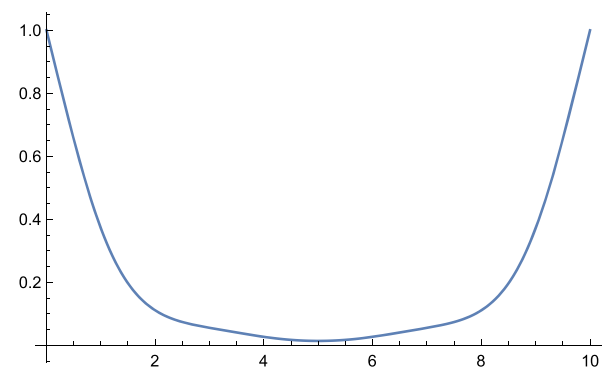

(a) $\Psi(r)$

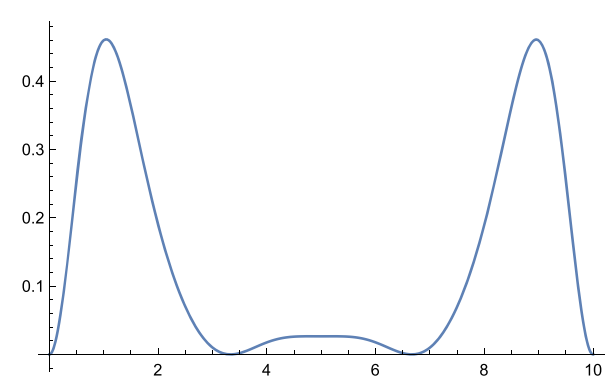

(c) $J_{\phi}(r)$

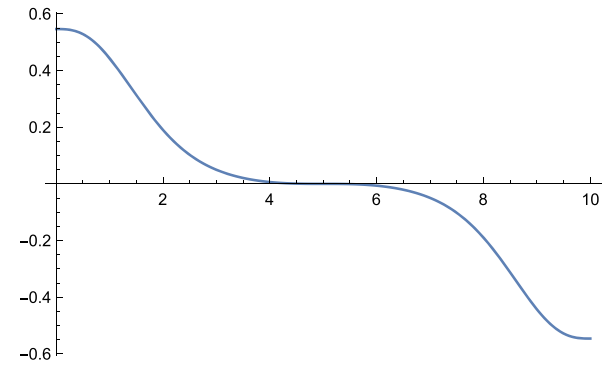

(b) $B(r)$

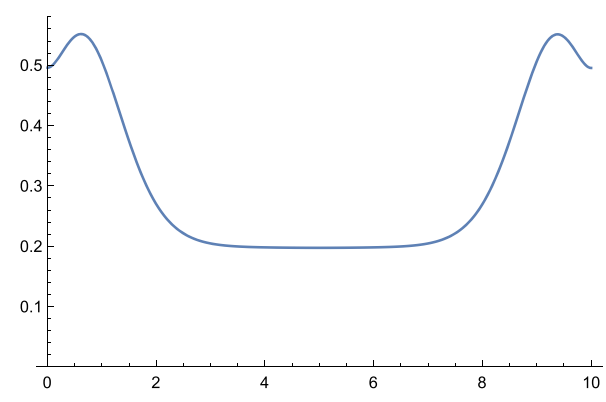

(d) $\rho(r)$

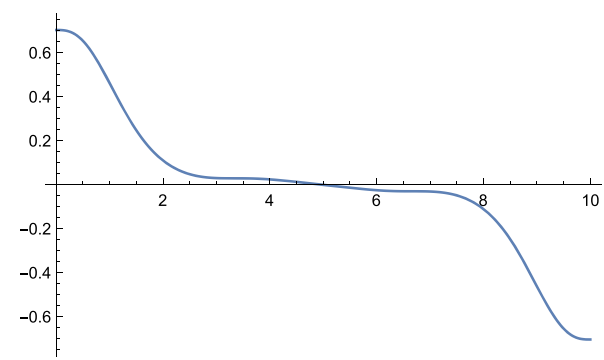

(b) $B(r)$

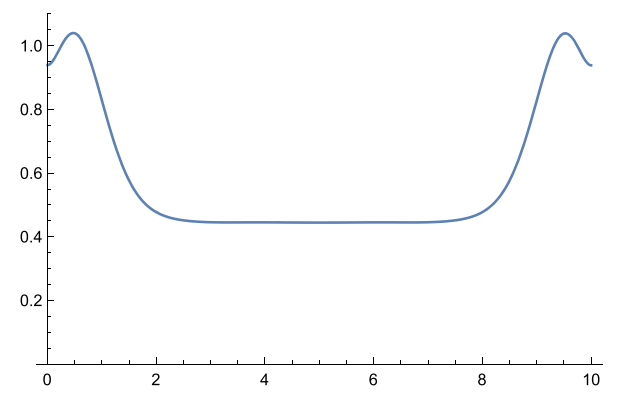

(d) $\rho(r)$ could be the "size" of the region in which the multi-solitonic configurations live, the soliton density, and so on. The correct resurgent parameter could be a combination of those. This situation should be compared with the many applications of resurgence in the literature in which the identification of the coupling constant is clean. An example which will be relevant for us is the Mathieu equation whose resurgent behavior has been deeply investigated (see [79] and references therein):

$$
\left(-\frac{\hbar^{2}}{2} \frac{\partial^{2}}{\partial x^{2}}+\cos x\right) \psi=E \psi
$$

In the above equation the only possible resurgent parameter is $\hbar$ while $E$ plays the role of an eigenvalue. 
Fig. 12 Profiles for the gauged baryon solution $n=0$ with anti-periodic boundaries for $\Psi$. The profile function $F(r)$ is defined by $E_{0}=\pi^{2} / 2 R^{2}$

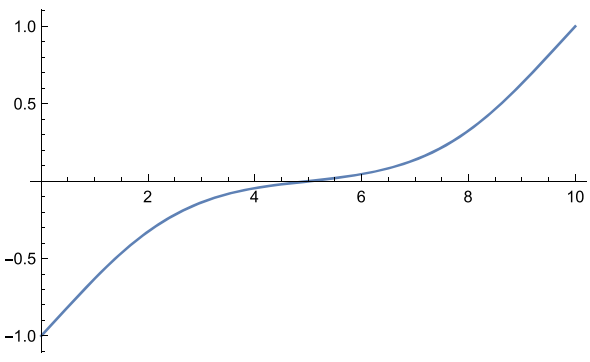

(a) $\Psi(r)$

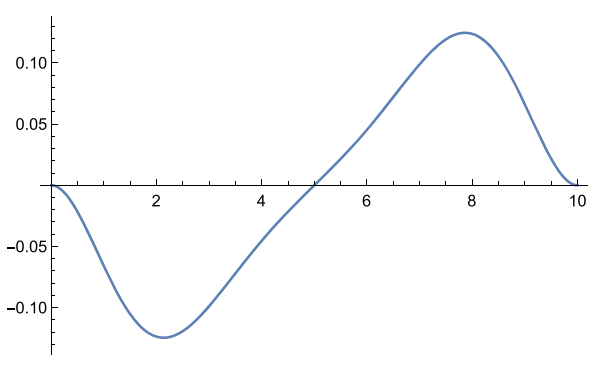

(c) $J_{\phi}(r)$

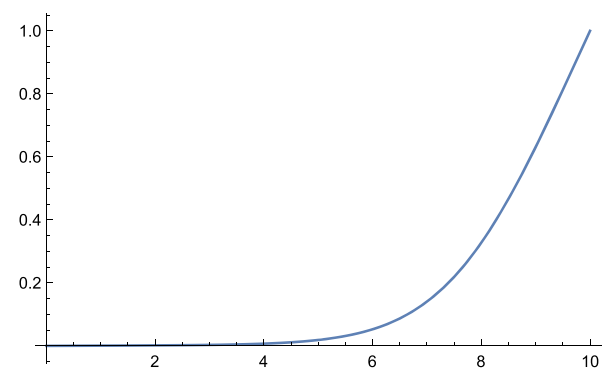

(a) $\Psi(r)$

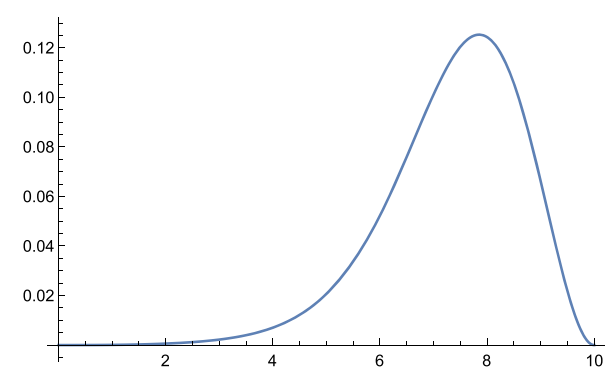

(c) $J_{\phi}(r)$

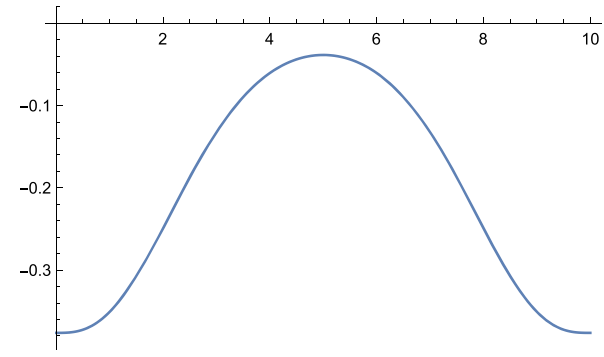

(b) $B(r)$

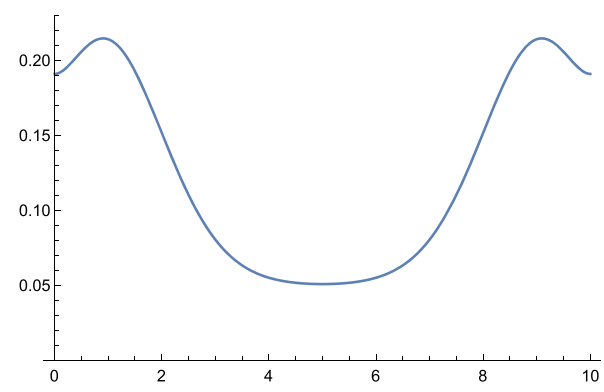

(d) $\rho(r)$

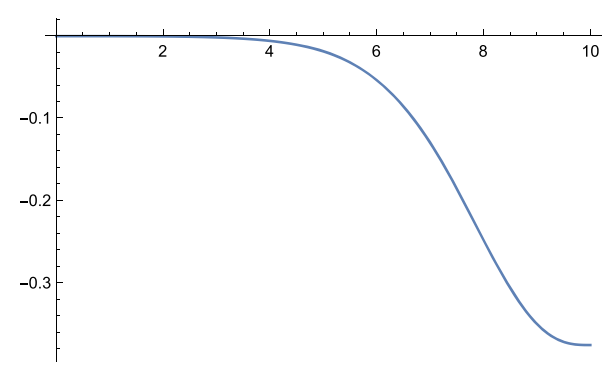

(b) $B(r)$

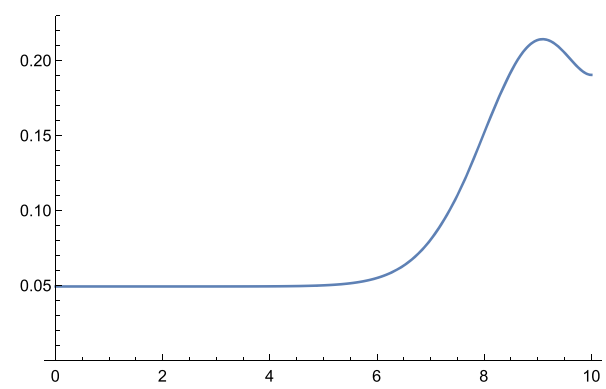

(d) $\rho(r)$
Here we show an explicit example of a solitonic system in which we are able to clearly identify the effective coupling constant that can be used for the resurgence study. This identification is possible comparing the baby Skyrme equations with the Mathieu equation (105).

The example of system that we analyse is the gauged baby Skyrmion discussed in the above section with $a_{0}=0$. Using a trigonometric equality, the Eq. (100) can be rewritten as

$$
\left[-\frac{\partial^{2}}{\partial r^{2}}+\frac{a_{1} g}{2} \cos \left(\frac{2 \pi}{R} r\right)\right] \Psi=\frac{a_{1} g}{2} \Psi .
$$

Now, let us define the new variable $x$ and the negative parameter $a_{1}$ as follows:

$$
\pi-x=\frac{2 \pi}{R} r \quad a_{1}=-\left|a_{1}\right| .
$$


Fig. 14 Profiles for the gauged baryon solution $n=0$ with non-vanishing potential $a_{0}=1$, using periodic boundaries for $\Psi$. The profile function $F(r)$ is defined by $E_{0}=0$

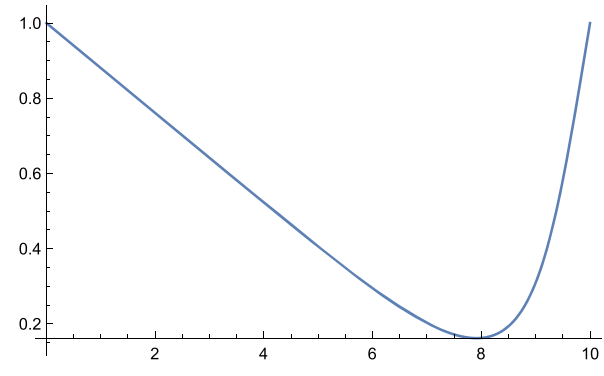

(a) $\Psi(r)$

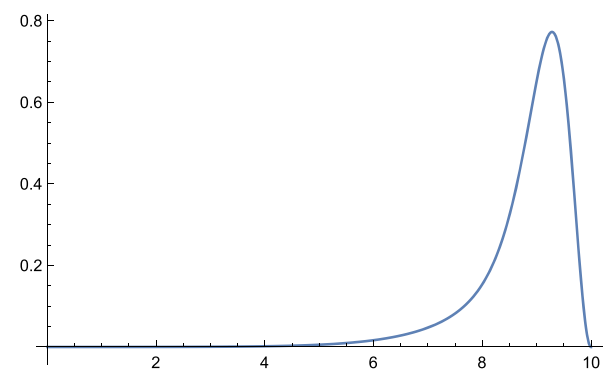

(c) $J_{\phi}(r)$

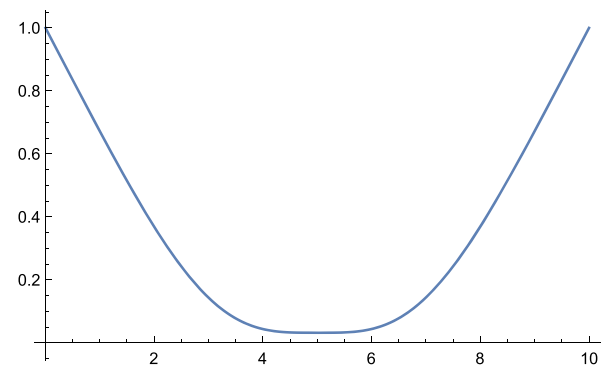

(a) $\Psi(r)$

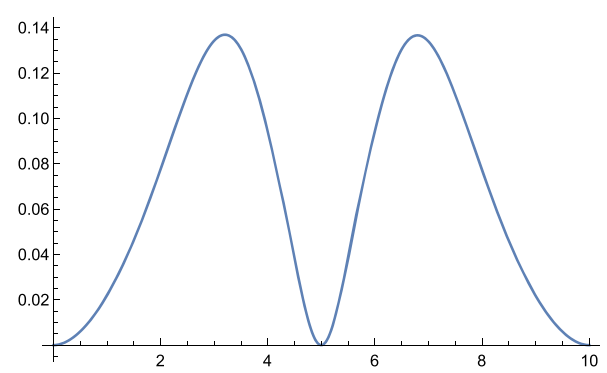

(c) $J_{\phi}(r)$

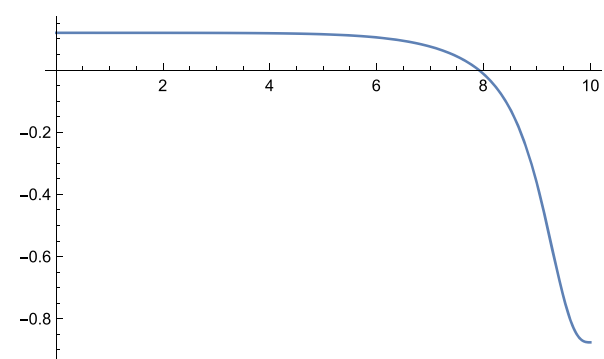

(b) $B(r)$

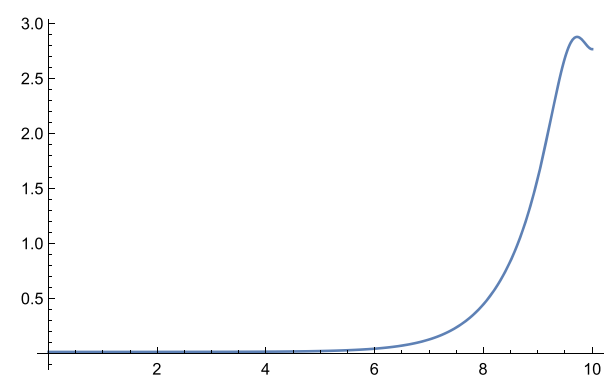

(d) $\rho(r)$

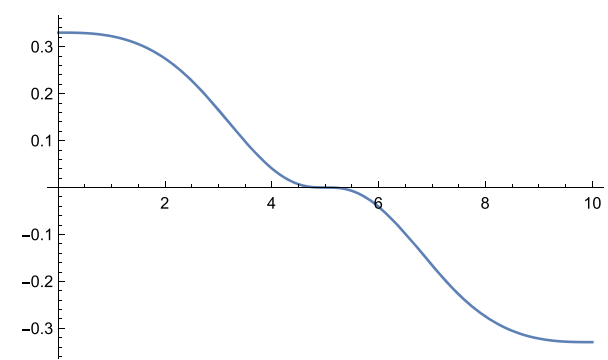

(b) $B(r)$

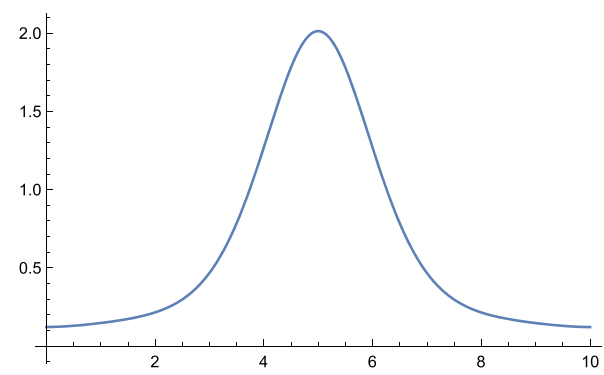

(d) $\rho(r)$
The remaining Maxwell equation (106) reduces to

$$
\left[-\left(\frac{8 \pi^{2}}{g\left|a_{1}\right| R^{2}}\right) \frac{\partial^{2}}{\partial x^{2}}+\cos (x)\right] \Psi=-\Psi .
$$

Hence, the above computations (together with a comparison with Eq. (105)) suggest that in this simplest non-trivial case the role of $\frac{\hbar^{2}}{2}$ is played by:

$$
\frac{\hbar^{2}}{2} \sim \frac{8 \pi^{2}}{g\left|a_{1}\right| R^{2}}
$$

Thus, in particular, the resurgent parameter is not only related to the "obvious" coupling constants $a_{1}$ and/or $a_{2}$ but actually is related with the suitable combination of both of them defined here above (which also involves the size of the system through $R$ ). It is worth to emphasize that only when analytic 
gauged solitonic solutions are available it is possible to discover the "proper coupling constant" (which, in the present case, is defined in Eq. (108)): this shows that the present analytic tools can be extremely useful from a resurgent perspective.

Equation (107) is a particular case of Eq. (105) analyzed, for instance, in [79] in which the eigenvalue has been fixed to -1 . On the other hand, the spectrum of the electromagnetic perturbations of these gauged solitons defined in Eqs. (76), (78), (98), (99) with $a_{0}=0$ in the gauged baby Skyrme model minimally coupled to Maxwell theory is closely related with the spectrum of the operator

$\widehat{O}=-\left(\frac{8 \pi^{2}}{g\left|a_{1}\right| R^{2}}\right) \frac{\partial^{2}}{\partial x^{2}}+\cos (x)$.

To prove this, it is sufficient to expand the field $\Psi$ around a background solution $\Psi_{0}$ (keeping $F$ and $G$ unchanged) as

$\Psi=\Psi_{0}+\delta \Psi \quad \delta \Psi=\sum c_{n} \delta \Psi_{n}$

where $\left\{\delta \Psi_{n}\right\}$ is a complete basis. In this case, the energy of the system with the electromagnetic perturbations reads

$E(\Psi)=E_{0}\left(\Psi_{0}\right)+\frac{1}{2} \sum\left|c_{n}\right|^{2} \lambda_{n}+\cdots$

where $\lambda_{n}$ is the set of eigenvalues of the operator

$\left[-\left(\frac{8 \pi^{2}}{g\left|a_{1}\right| R^{2}}\right) \frac{\partial^{2}}{\partial x^{2}}+\cos (x)+1\right] \delta \Psi_{n}=\lambda_{n} \delta \Psi_{n}$.

This operator is simply calculated linearizing equation (107). Defining for simplicity $\lambda_{n}^{\prime}=\lambda_{n}-1$, we obtain exactly the Mathieu equation (105) with the effective resurgence parameter.

Thus, quite interestingly, the full resurgent analysis of the Mathieu equation in [79] is relevant to determine the spectrum of the operator $\widehat{O}$ which, in its turn, plays an important role to determine the (spectrum of the) electromagnetic perturbations of these analytic gauged solitons. We hope to come back on this interesting issue on the resurgent analysis of the perturbations of these gauged solitons in a future publication.

One may also ask what happens in the more general cases in which the potential term in Eq. (85) does not vanish $a_{0} \neq 0$. In this case, the profile can be integrated explicitly in terms of the quadrature in Eq. (86). When $k=1$ or $k=2$, the quadrature in Eq. (86) is explicitly solved in terms of inverse elliptic functions (Jacobi Amplitude functions [125], call them $A(r)$ ). Thus, the remaining Maxwell equation in Eq. (88) looks similar to a deformed Mathieu equation with the differences that, firstly, the argument " $r$ " of the trigonometric function has been replaced with $A(r)$ and, secondly, the coupling constant $a_{1} g$ has been replaced by a new function involving the derivative of $A(r)$. Schematically, one gets:

$$
\left\{\left(\frac{\partial^{2}}{\partial r^{2}}+a_{1} g \sin ^{2}\left(\frac{\pi}{R} r\right)\right) \Psi=0\right\} \Rightarrow
$$

$$
\left(\frac{\partial^{2}}{\partial r^{2}}+a_{1}\left(1-\left(A^{\prime}\right)^{2}\right) \sin ^{2}(A(r))\right) \Psi=0 .
$$

Thus, besides the explicit appearance of the coupling constants as in the previous simpler case, now the potential depends in a more implicit way on the boundary condition (through the integration constant appearing in the function $J(r)$ ). We hope to come back in a future publication on the resurgent analysis of these Schrodinger-like problems arising in the description of the multi-solitonic solutions of the gauged baby Skyrme model in (2+1)-dimensions.

\subsection{Persistent residual currents}

Another interesting characteristic of the gauged baby Skyrmions, which is valid also in the present case, is that they generate a current with many features in common with superconductive currents. Let us consider, once again, the simplest non-trivial case in the previous subsection of Eqs. (98), (99) in which $a_{0}=0$ (no potential term). One can notice the following characteristic of the current in Eq. (90).

(1) The current does not vanish even when the electromagnetic potential vanishes $\left(A_{\mu}=0\right)$.

(2) This left-over current $J_{(0) \mu}$

$$
\begin{aligned}
J_{(0) \mu}= & J_{\mu_{A_{\mu}=0}} \\
= & -\left[a_{1} \sin ^{2}(F)\left(\nabla_{\mu} G\right)-a_{2} \sin ^{2}(F)\right. \\
& \left.\times\left(\left(\nabla^{\nu} F \nabla_{\nu} F\right)\left(\nabla_{\mu} G\right)-\left(\nabla^{\nu} F \nabla_{\nu} G\right) \nabla_{\mu} F\right)\right] \\
= & -a_{1} \sin ^{2}\left(\frac{\pi}{R} r\right)\left[1-\frac{a_{2}}{a_{1}}\left(\frac{\pi}{R}\right)^{2}\right]\left(\nabla_{\mu} G\right)
\end{aligned}
$$

cannot be turned off continuously. One can try to eliminate $J_{(0) \mu}$ either deforming $F$ to integer multiples of $\pi$ (but this is impossible as such a deformation would kill the topological charge as well: see Eq. (13)) or deforming $G$ to a constant (but also this deformation cannot be achieved for the same reason). Note also that, as happens in the construction of superconducting currents in [93], $G$ is defined modulo $2 \pi$ (as $\vec{\Phi}$ depends on $\cos G$ and $\sin G$ rather than on $G$ itself). This implies that $J_{(0) \mu}$ defined in Eq. (112) is a persistent current supported by the present gauged baby Skyrmions. Needless to say, the persistent character of a current is the cleanest hallmark of superconductivity. Moreover, just recently in [80,81], the first glimpse of resurgent structures appeared in integrable many body models of superconductivity. The present results open the intriguing possibility to analyze superconductive currents with the tools of resurgence in a nonintegrable model such as the gauged baby Skyrme model in $(2+1)$ dimensions. 
A final remark on the stability of these solutions has to be made. The eigenvalue analysis presented above for the ungauged case is applicable in this case aswell, with the extensive complexification of having to deal with all types of gauge perturbations possible. This type of analysis is computationally hard to achieve as the size of the pertubation operator becomes large. If we reduce the space of perturbations to only the ones deforming the field profiles as we did above, then we expect a similar stability analysis resulting in no negative eigenvalues. The full perturbation space is however much larger and we leave this full stability analysis as an open problem.

\section{Conclusions}

Analytic topologically non-trivial solutions of the complete set of baby Skyrme field equations of arbitrary charge living at finite density have been constructed explicitly. These solutions describe an alternating sequence of baby skyrmions and anti-baby skyrmions located along the cylinder: the babybaryon density shows a multi-peaks structure. We call these configurations topological solitons dressed by kinks. These new kinds of solutions are characterized by two integers: $n$ (or $m$ ) (which is related to the number of bumps in the baryon density, i.e. the number of baby skyrmions within the cylinder) and $p$ (which is related to the baryon charge of each single baby skyrmion). The configurations characterized by $m$ are periodic in the field and in the first derivative of the field and therefore they represent the cell of a physical smooth crystal. Otherwise, the solutions identified by the integer $n$ describe $(2 n+1)$-ordered peaks of baryon matter located at a finite space. All these configurations pass a non-trivial stability test. Moreover, the first analytic examples of gauged baby Skyrmions with nonvanishing topological charge in (2+1)-dimensions in the gauged baby Skyrme model, minimally coupled to Maxwell theory, have been constructed. The complete set of 5 coupled non-linear field equations can be reduced (using a judiciously chosen ansatz) in a self-consistent way to one linear Schrodinger-like equation with an effective periodic potential keeping alive, at the same time, the baby baryonic charge. These analytic gauged baby Skyrmions generate a persistent $U(1)$ current which cannot be turned off continuously as it is tied to the topological charge of the baby Skyrmions themselves. It appears that the present gauged multi-Solitonic configurations are a very suitable arena to test resurgence in a non-integrable context as these solitons allow a clear identification of the proper coupling constant which is responsible for the resurgent behavior.

Acknowledgements F. C. and G. T. have been funded by Fondecyt Grants 1200022 and 1200025. The Centro de Estudios Científicos
(CECs) is funded by the Chilean Government through the Centers of Excellence Base Financing Program of Conicyt. The work of M. B. and $\mathrm{S}$. B. is supported by the INFN special project grant "GAST (Gauge and String Theories)".

Data Availability Statement This manuscript has no associated data or the data will not be deposited. [Authors' comment: Data sharing not applicable to this article as no datasets were generated or analysed during the current study.]

Open Access This article is licensed under a Creative Commons Attribution 4.0 International License, which permits use, sharing, adaptation, distribution and reproduction in any medium or format, as long as you give appropriate credit to the original author(s) and the source, provide a link to the Creative Commons licence, and indicate if changes were made. The images or other third party material in this article are included in the article's Creative Commons licence, unless indicated otherwise in a credit line to the material. If material is not included in the article's Creative Commons licence and your intended use is not permitted by statutory regulation or exceeds the permitted use, you will need to obtain permission directly from the copyright holder. To view a copy of this licence, visit http://creativecomm ons.org/licenses/by/4.0/.

Funded by $\mathrm{SCOAP}^{3}$.

\section{References}

1. T. Skyrme, Proc. R. Soc. Lond. A 260, 127 (1961)

2. T. Skyrme, Proc. R. Soc. Lond. A 262, 237 (1961)

3. T. Skyrme, Nucl. Phys. 31, 556 (1962)

4. C.G. Callan Jr., E. Witten, Nucl. Phys. B 239, 161-176 (1984)

5. E. Witten, Nucl. Phys. B 223, 422 (1983)

6. E. Witten, Nucl. Phys. B 223, 433 (1983)

7. A.P. Balachandran, V.P. Nair, N. Panchapakesan, S.G. Rajeev, Phys. Rev. D 28, 2830 (1983)

8. A.P. Balachandran, A. Barducci, F. Lizzi, V.G.J. Rodgers, A. Stern, Phys. Rev. Lett. 52, 887 (1984)

9. A.P. Balachandran, F. Lizzi, V.G.J. Rodgers, A. Stern, Nucl. Phys. B 256, 525-556 (1985)

10. G.S. Adkins, C.R. Nappi, E. Witten, Nucl. Phys. B 228, 552-566 (1983)

11. B. Piette, B. Schroers, W. Zakrzewski, Nucl. Phys. B 439, 205 235 (1995)

12. S. Bolognesi, W. Zakrzewski, Phys. Rev. D 91(4), 045034 (2015)

13. N. Manton, P. Sutcliffe, Topological Solitons (Cambridge University Press, Cambridge, 2007)

14. A. Balachandran, G. Marmo, B. Skagerstam, A. Stern, Classical Topology and Quantum States (World Scientific, Singapore, 1991)

15. B.J. Schroers, Phys. Lett. B 356, 291-296 (1995)

16. K. Arthur, D.H. Tchrakian, Phys. Lett. B 378, 187-193 (1996)

17. J. Gladikowski, B.M.A.G. Piette, B.J. Schroers, Phys. Rev. D 53, 844 (1996)

18. Y. Brihaye, D.H. Tchrakian, Nonlinearity 11, 891 (1998)

19. AYu. Loginov, V.V. Gauzshtein, Phys. Lett. B 784, 112-117 (2018)

20. C. Adam, C. Naya, J. Sanchez-Guillen, A. Wereszczynski, Phys. Rev. D 86, 045010 (2012)

21. C. Adam, C. Naya, J. Sanchez-Guillen, A. Wereszczynski, Phys. Rev. D 95(4), 045002 (2017)

22. C. Adam, J.M. Queiruga, J. Sanchez-Guillen, A. Wereszczynski, JHEP05 108 (2013)

23. C. Adam, C. Naya, T. Romanczukiewicz, J. Sanchez-Guillen, A. Wereszczynski, JHEP 05, 155 (2015) 
24. C. Adam, T. Romanczukiewicz, J. Sanchez-Guillen, A. Wereszczynski, JHEP 11, 095 (2014)

25. A. Alonso-Izquierdo, W. Garcia Fuertes, JHEP 02, 139 (2015)

26. A. Samoilenka, Ya. Shnir, Phys. Rev. D 93, 065018 (2016)

27. S. Chimento, T. Ortin, A. Ruiperez, JHEP 05, 107 (2018)

28. P. de Forcrand, Simulating QCD at finite density, PoS(LAT2009)010. arXiv:1005.0539 [INSPIRE]

29. N. Brambilla et al., QCD and strongly coupled gauge theories: challenges and perspectives. Eur. Phys. J. C 74, 2981 (2014). arXiv: 1404.3723 [INSPIRE]

30. Y. Nambu, G. Jona-Lasinio, Phys. Rev. 122, 345 (1961)

31. Y. Nambu, G. Jona-Lasinio, Phys. Rev. 124, 246 (1961)

32. K. Rajagopal, F. Wilczek, in At the Frontier of Particle Physics/Handbook of QCD, ed. by M. Shifman (World Scientific, Singapore, 2001). arXiv:hep-ph/0011333

33. M.G. Alford, J.A. Bowers, K. Rajagopal, Phys. Rev. D 63, 074016 (2001)

34. R. Casalbuoni, G. Nardulli, Rev. Mod. Phys. 76, 263 (2004)

35. A.I. Larkin, YuN Ovchinnikov, Zh Eksp, Teor. Fiz. 47, 1136 (1964)

36. A.I. Larkin, Y.N. Ovchinnikov, Sov. Phys. JETP. 20, 762

37. P. Fulde, R.A. Ferrell, Phys. Rev. 135, A550 (1964)

38. L. McLerran, R.D. Pisarski, Nucl. Phys. A 796, 83 (2007)

39. Y. Hidaka, L.D. McLerran, R.D. Pisarski, Nucl. Phys. A 808, 117 (2008)

40. L.Y. Glozman, R.F. Wagenbrunn, Phys. Rev. D 77, 054027 (2008)

41. D.J. Gross, A. Neveu, Phys. Rev. D 10, 3235 (1974)

42. R.F. Dashen, B. Hasslacher, A. Neveu, Phys. Rev. D 12, 2443 (1975)

43. S.S. Shei, Phys. Rev. D 14, 535 (1976)

44. J. Feinberg, A. Zee, Phys. Rev. D 56, 5050 (1997)

45. G. Basar, G.V. Dunne, Phys. Rev. Lett. 100, 200404 (2008)

46. G. Basar, G.V. Dunne, Phys. Rev. D 78, 065022 (2008)

47. G. Basar, G.V. Dunne, M. Thies, Phys. Rev. D 79, 105012 (2009)

48. V. Schon, M. Thies, Phys. Rev. D 62, 096002 (2000)

49. V. Schon, M. Thies, in At the Frontier of Particle Physics, vol. 3, ed. by M. Shifman (World Scientific, Singapore, 2001)

50. M. Thies, Phys. Rev. D 69, 067703 (2004)

51. M. Thies, J. Phys. A 39, 12707 (2006)

52. B. Bringoltz, J. High Energy Phys. 03, 016 (2007). arXiv:0901.4035

53. D. Nickel, M. Buballa, Phys. Rev. D 79, 054009 (2009)

54. D. Nickel. arXiv:0902.1778

55. F. Karbstein, M. Thies, Phys. Rev. D 75, 025003 (2007)

56. K. Takayama, M. Oka, Nucl. Phys. A 551, 637-656 (1993)

57. T. Brauner, N. Ya mamoto, JHEP 1704, 132 (2017)

58. X.G. Huang, K. Nishimura, N. Yamamoto, JHEP 1802, 069 (2018)

59. M. Buballa, S. Carignano, Prog. Part. Nucl. Phys. 81, 39 (2015)

60. K. Splittorff, D.T. Son, M.A. Stephanov, Phys. Rev. D 64, 016003 (2001)

61. D.G. Ravenhall, C.J. Pethick, J.R. Wilson, Phys. Rev. Lett. 50, 2066 (1983)

62. M. Hashimoto, H. Seki, M. Yamada, Prog. Theor. Phys. 71, 320 (1984)

63. C.J. Horowitz, D.K. Berry, C.M. Briggs, M.E. Caplan, A. Cumming, A.S. Schneider, Phys. Rev. Lett. 114, 031102 (2015)

64. D.K. Berry, M.E. Caplan, C.J. Horowitz, G. Huber, A.S. Schneider, Phys. Rev. C 94, 055801 (2016)

65. M. Marino, Instantons and Large N: an introduction to Nonperturbative methods in Quantum Field Theory (Cambridge University Press, Cambridge, 2015)

66. J.C. Le Guillou, J. Zinn-Justin, Large order behavior of perturbation theory (North-Holland, Amsterdam, 1990)

67. M.C. Berry, C.J. HoDls, Proc. R. Soc. A 430, 653 (1990)

68. M.C. Berry, C.J. HoDls, Proc. R. Soc. E 434, 657 (1991)
69. E.M.C. Berry, Asymptotics, superasymptotics, hyperasymptotics, in Asymptotics beyond all orders, ed. by H. Segur, et al. (Plenum Press, New York, 1991)

70. I. Aniceto, G. Basar, R. Schiappa, A primer on resurgent transseries and their asymptotics. arXiv: 1802.10441

71. E.B. Bogomolny, Phys. Lett. B 91, 431 (1980)

72. J. Zinn-Justin, Nucl. Phys. B 192, 125 (1981)

73. M. Marino, R. Schiappa, M. Feiss, Commun. Num. Theor. Phys. 2, 349 (2008)

74. M. Marino, R. Schiappa, M. Feiss, E J. Math. Phys. 50, 052301 (2009)

75. S. Demulder, D. Dorigoni, D.C. Thompson, J. High Energy Phys. 07, 088 (2016)

76. G. Basar, G.C. Dunne, J. High Energy Phys. 02, 160 (2015)

77. I. Aniceto, R. Schiappa, Comm. Math. Phys. 335, 183-245 (2015)

78. I. Aniceto, J. Russo, R. Schiappa, JHEP 1503, 172 (2015)

79. G.C. Dunne, M. Unsal, in Resurgence, Physics and Numbers, Edizioni Della Normale (2017). arXiv:1603.04924

80. M. Marino, T. Reis, J. Stat. Mech. 123102 (2019)

81. M. Marino, T. Reis, J. Stat. Phys. https://doi.org/10.1007/ s10955-019-02413-1

82. F. Canfora, G. Tallarita, Phys. Rev. D 94(2), 025037 (2016)

83. G. Tallarita, F. Canfora, Nucl. Phys. B B921, 394-410 (2017)

84. P.D. Alvarez, F. Canfora, N. Dimakis, A. Paliathanasis, Phys. Lett. B 773, 401-407 (2017)

85. L. Aviles, F. Canfora, N. Dimakis, D. Hidalgo, Phys. Rev. D 96, 125005 (2017)

86. F. Canfora, M. Lagos, S.-H. Oh, J. Oliva, A. Vera, Phys. Rev. D 98(8), 085003 (2018)

87. F. Canfora, Eur. Phys. J. C 78(11), 929 (2018)

88. F. Canfora, S.-H. Oh, A. Vera, Eur. Phys. J. C 79(6), 485 (2019)

89. F. Canfora, M. Lagos, A. Vera, Eur. Phys. J. C 80(8), 697 (2020)

90. P.D. Alvarez, S.L. Cacciatori, F. Canfora, B.L. Cerchiai, Phys. Rev. D 101(12), 125011 (2020)

91. E. Ayón-Beato, F. Canfora, M. Lagos, J. Oliva, A. Vera, Eur. Phys. J. C 80(5), 384 (2020)

92. F. Canfora, A. Giacomini, M. Lagos, S.-H. Oh, A. Vera, Gravitating cosmic superconducting tubes in the Einstein gauged nonlinear sigma-model in (3+1)-dimensions. arXiv:2001.11910

93. E. Witten, Nucl. Phys. B 249, 557 (1985)

94. M. Shifman, Advanced topics in quantum field theory: a lecture course (Cambridge University Press, Cambridge, 2012)

95. A. Vilenkin, E.P.S. Shellard, Cosmic strings and other topological defects (Cambridge University Press, Cambridge, 1994)

96. R.L. Davis, E.P.S. Shellard, Cosmic Vortons. Nucl. Phys. B 323, 209 (1989)

97. R.H. Brandenberger, B. Carter, A.C. Davis, M. Trodden, Phys. Rev. D 54, 6059 (1996)

98. A.C. Davis, W.B. Perkins, Phys. Lett. B 393, 46 (1997)

99. L. Masperi, M. Orsaria, Int. J. Mod. Phys. A 14, 3581 (1999)

100. L. Masperi, G.A. Silva, Astropart. Phys. 8, 173 (1998)

101. E. Radu, M.S. Volkov, Phys. Rept. 468, 101 (2008)

102. Y. Lemperiere, E.P.S. Shellard, Phys. Rev. Lett. 91, 141601 (2003)

103. Y. Lemperiere, E.P.S. Shellard, Nucl. Phys. B 649, 511 (2003)

104. J. Garaud, E. Radu, M.S. Volkov, Phys. Rev. Lett. 111, 171602 (2013)

105. M. Shifman, Phys. Rev. D 87(2), 025025 (2013)

106. B.M.A.G. Piette, D.H. Tchrakian, Phys. Rev. D 62, 025020 (2000)

107. A. Al-Zamel, V.K. Tuan, S.L. Kalla, Appl. Math. Comput. 114(13), 25 (2000)

108. Mridula Garg, Vimal Katta, S. Kalla, Serd. Math. J. 27(3), 219232 (2001)

109. M. Garg, V. Katta, S.L. Kalla, Appl. Math. Comput. 131(607), 613 (2002) 
110. F. Arscott, Periodic differential equations: an introduction to Mathieu, Lamé, and allied functions (Pergamon Press, New York, 1964)

111. J.C. Gutiérrez-Vega, Mathieu Functions, in The Princeton Companion to Applied Mathematics, ed. by Nicholas J. Higham, et al. (Princeton University Press, Princeton, 2015), pp. 159-160

112. P. Malits, Relations between Mathieu functions of the first and second kind. Integral Transforms Spec Funct. 21(6), 423-436 (2010)

113. K. Takayama, M. Oka, Nucl. Phys. A 551, 637-656 (1993)

114. V. Schön, M. Thies, Phys. Rev. D 62, 096002 (2000)

115. S. Bolognesi, P. Sutcliffe, J. Phys. A 47, 135401 (2014)

116. D.G. Ravenhall, C.J. Pethick, J.R. Wilson, Phys. Rev. Lett. 50, $2066(1983)$

117. M. Hashimoto, H. Seki, M. Yamada, Prog. Theor. Phys. 71, 320 (1984)

118. C.J. Horowitz, D.K. Berry, C.M. Briggs, M.E. Caplan, A. Cumming, A.S. Schneider, Phys. Rev. Lett. 114, 031102 (2015)

119. D.K. Berry, M.E. Caplan, C.J. Horowitz, G. Huber, A.S. Schneider, Phys. Rev. C 94, 055801 (2016)
120. M. Shifman, Advanced Topics in Quantum Field Theory: A Lecture Course (Cambridge University Press, Cambridge, 2012)

121. M. Shifman, A. Yung, Supersymmetric Solitons (Cambridge University Press, Cambridge, 2009)

122. H. Weigel, Chiral Soliton Models for Baryons, Lecture Notes in Physics (Springer, Berlin, 2008)

123. A. Al-Zamel, V.K. Tuan, S.L. Kalla, Appl. Math. Comput. 114, $13-25(2000)$

124. M. Garg, V. Katta, S.L. Kalla, Appl. Math. Comput. 131, 607-613 (2002)

125. M. Abramowitz, I. Stegun, Handbook of Mathematical Functions with Formulas, Graphs, and Mathematical Tables. Applied Mathematics Series. 55 (Dover, (1983) Chapter 16)

126. D. Harland, R.S. Ward, Walls and chains of planar skyrmions. Phys. Rev. D 77, 045009 (2008) 